\title{
Nonlinear dynamo action in rotating convection and shear
}

\author{
By PU ZHANG, ANDREW D. GILBERT AND KEKE ZHANG
}

Department of Mathematical Sciences, University of Exeter, Exeter EX4 4QE, UK

(Received 24 August 2004 and in revised form 16 June 2005)

Magnetic field amplification by the motion of an electrically conducting fluid is studied, using a rotating plane-layer geometry. The fluid flow is driven by convection, and by a moving bottom boundary, which leads to an Ekman layer localized at the base of the system. The system thus has the structure of an interface dynamo, with convection lying over a thin layer of shear.

The combination of shear in the Ekman layer and convection above leads to amplification of seed magnetic fields. In kinematic regimes the magnetic field is mostly localized in sheets in the shear layer, but thin tongues are pulled out by the convection above and folded. The nonlinear saturation of these growing fields is studied at moderately high values of magnetic Reynolds number and Taylor number. It is found that the sheets of field tend to gain fine-scale structure when the dynamo saturates, breaking up into tubes, and the fluid flow shows complex time-dependence. Although the magnetic field lies predominantly within the highly sheared Ekman layer, this flow remains remarkably unchanged despite the action of Lorentz forces. Instead, the effect of the field is to suppress or modify the convection above. A simple alpha-omega dynamo model is set up, and gives some insights into the dynamo processes occurring in the full magnetohydrodynamic simulation.

\section{Introduction}

Astrophysical, geophysical and planetary dynamos are dominated by processes of convection and shear. Our aim in this paper is to study the nonlinear evolution and saturation of magnetic fields in an interface dynamo, with shear and thermal convection, relevant to understanding the generation of the Sun's magnetic field. The early modelling of the solar dynamo (see, for example, Moffatt 1978; Parker 1979; Krause \& Rädler 1980) included the effects of shear and convection, modelled by an $\alpha$-effect, and showed how dynamo waves can be generated by an $\alpha \omega$ dynamo mechanism. With the correct signs for the shear and $\alpha$-effect, the waves propagate from poles to equator in agreement with solar observations.

This view ran into problems when large-scale numerical simulations of rotating convection in a spherical shell (Gilman 1983; Glatzmaier 1984; 1985a,b) gave robust poleward migration of magnetic fields. A few years later, helioseismological results (Brown et al. 1989; Dziembowski, Goode \& Libbrecht 1989; Goode et al. 1991; Gough, Sekii \& Stark 1996) revealed that the angular velocity profile of the Sun includes a thin layer of shear in a region between the inner radiative zone and the outer convection zone. This thin layer of shear is known as the tachocline (Spiegel \& Zahn 1992) and may indicate the presence of a relict magnetic field within the 
radiative zone, decoupled from the dynamo-generated field that we observe (Gough \& McIntyre 1998; Garaud 2002).

The discovery of the tachocline led to the idea that the Solar magnetic field may be generated in this strong layer of shear, which would provide an $\omega$-effect (e.g. Roberts 1992; Weiss 1994). There are a number of possible ways in which an $\alpha$-effect could arise to complete the dynamo cycle. These include convection, hydrodynamical instability (e.g. Dikpati \& Gilman 2001) and magnetic buoyancy (e.g. Thelen 2000). From this point, one approach is to parameterize the three-dimensional processes that give an $\alpha$-effect and turbulent diffusivities, obtaining butterfly diagrams that can be compared with solar data (Deluca \& Gilman 1986, 1988; Parker 1993; Prautzsch 1993; Rüdiger \& Brandenburg 1995; Tobias 1996; Roald 1998; Tobias, Proctor \& Knobloch 1998; Griffiths et al. 2001; Zhang et al. 2003). Alternatively, one can simulate a simplified magnetohydrodynamic system by means of three-dimensional simulations, and understand its behaviour in various regimes. This has the advantage that primitive equations are employed, and so avoids the uncertainty in parameterizing complex turbulent transport effects, the disadvantage being that the parameter values obtainable are far from those pertaining to the Sun. We will adopt this second approach in this paper, and set up a model based on convection in the presence of rotation and a thin layer of shear.

There have been many studies of dynamos in rotating convective flows, beginning with Childress \& Soward (1972), who showed that an $\alpha$-effect can be obtained asymptotically at low magnetic Reynolds numbers and give growth of large-scale magnetic fields. Matthews (1998) further showed that kinematic dynamo action can occur in weakly nonlinear regimes with fields being amplified on the scale of the flow, at large magnetic Reynolds numbers. Simulations by St Pierre (1993), Jones \& Roberts (2000) and Rotvig \& Jones (2002) have pushed these results into strongly nonlinear, turbulent regimes, in the limit of small Ekman number. These studies adopted a rotating plane-layer geometry. A different approach was pioneered by Busse (1975) which is to incorporate elements of spherical geometry in a rotating annulus model with inclined top and bottom surfaces. This allows Rossby waves to be driven by convection, and dynamo action in such a flow was studied by Kim, Hughes \& Soward $(1999,2004)$. Other relevant simulations include dynamo action in convection over a region of stable stratification (Brandenburg et al. 1990; Nordlund et al. 1992; Tobias et al. 2001), in accretion disks dominated by Keplerian shear and hydromagnetic instability (Brandenburg et al. 1995), in convective flows in a rapidly rotating sphere (Busse 2002) and in shear with magnetic buoyancy providing an $\alpha$-effect (Cline, Brummell \& Cattaneo 2003).

The aim in this paper is to study a fully hydrodynamical interface dynamo in a plane-layer geometry by means of three-dimensional numerical simulations. The model was introduced in Ponty, Gilbert \& Soward (2001, hereinafter referred to as paper I), and is motivated as a classical fluid flow with some features in common with the solar tachocline and the convection zone just above it. A plane-layer geometry is adopted, based on an approximation to spherical geometry at a co-latitude $\vartheta$, as depicted in figure 1 . The plane layer is subjected to rotation and heating, leading to thermally driven convection. The additional feature present is that the bottom boundary has a velocity $U_{0}$ as shown and, with no-slip boundary conditions, this gives an Ekman-Couette shear flow across the plane layer. In the limit of strong rotation, this flow becomes an Ekman layer localized at the bottom boundary. There is a corresponding Ekman flux in the perpendicular direction (which in the solar context would be returned as part of a meridional circulation at higher $z$, i.e. greater 

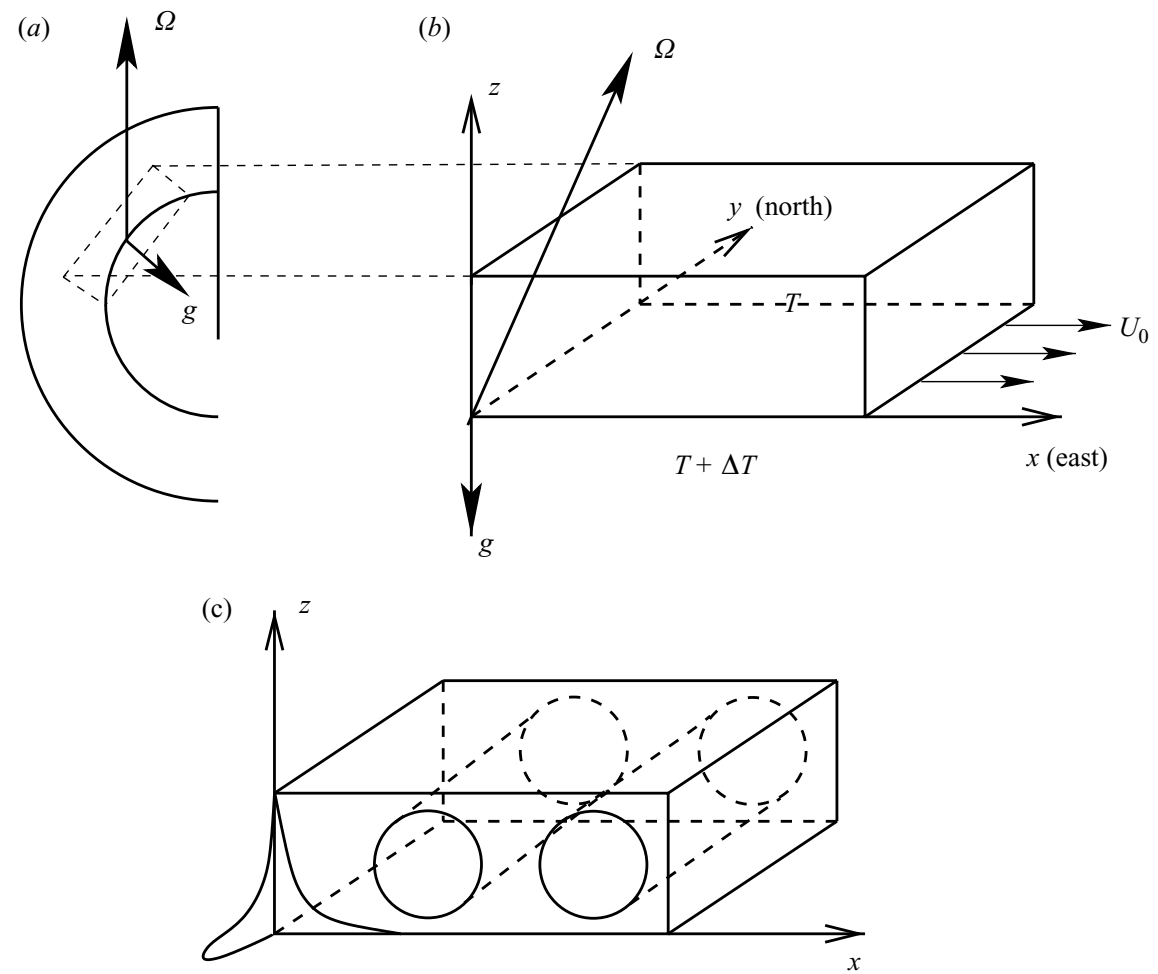

FigurE 1. Geometry for the study. (a) Spherical shell geometry is approximated as (b) plane-layer geometry. (c) Schematic picture of the fluid flow with an Ekman layer at the base, and convective rolls in the interior.

radii). Note that there is no Ekman boundary layer generated at the top boundary; the horizontal pressure gradient, which is zero in our frame of reference, is not Galilean invariant in a rotating reference frame.

This hydrodynamical system allows a rich array of instabilities, including TaylorCouette, convective, and Ekman instabilities, as discussed in Matthews \& Cox (1997), Hoffman, Busse \& Chen (1998) and Ponty, Gilbert \& Soward (2003). These can lead to flows sufficiently complex to give dynamo action, and this was explored numerically in paper I, in the kinematic regime, with emphasis on the limit of large magnetic Reynolds number. The flows were taken to be two-dimensional, depending on two coordinates, which is correct at the onset of instability, and allowed the consideration of magnetic modes with a wavenumber $k$ in a perpendicular direction. The paper first explored dynamo action in flows resulting from equilibrated Ekman instabilities, which occur when a Reynolds number based on the thickness of the Ekman layer is sufficiently large. The resulting secondary fluid flow lies predominantly within the Ekman layer with roll axes approximately aligned with the $x$-axis, that is East-West for the geometry in figure 1. The flows give dynamo action, with magnetic fields taking the form of Ponomarenko modes, spiralling tubes of field in a convective cell, or separatrix modes, sheets of field localized at hyperbolic stagnation points and along heteroclinic connections. These are slow dynamo modes (e.g. Childress \& Gilbert 1995; Gilbert 2003) as the flow is steady in a translating frame and twodimensional. 
For the second class of flows studied in paper I, parameter values were used where the Ekman layer is hydrodynamically stable, but the fluid is convectively unstable. The convective rolls generated tend to be excluded from the Ekman layer, and lie above it with their axes approximately aligned with the $y$-axis, that is North-South. This gives a natural interface dynamo: convection above a thin layer of shear. The magnetic fields generated have a strong component within the shear layer, and undergo slow dynamo action when the flow is steady (in a translating frame) and two-dimensional, and fast dynamo action (with sheets of field accumulating in regions of chaotic particle paths) when the flow becomes unsteady (in every reference frame).

In this paper, we extend our study into nonlinear regimes. We focus on the case of convective instability only; the case of Ekman instability will be the focus of a future study. Our interest is in the saturation of the magnetic fields in the convective dynamos identified in paper I. As discussed above, this gives a natural interface dynamo, resulting from a classical hydrodynamic geometry, with no assumptions about transport coefficients, and our aim is to understand fundamental issues about the nature of equilibration by means of numerical simulations.

Since the code we use is fully three-dimensional, we are not able to explore parameter space in much detail, and so we have to focus on a few key issues. The first issue is the nature of dynamo saturation: does the magnetic field disrupt the layer of shear, or modify the convection, or some combination, when it equilibrates? Secondly, what happens to the magnetic field in nonlinear regimes: does it adopt fine-scale structure, on the Ekman-layer scale, or does it prefer to seek the largest scale in the system? Thirdly, to what extent can we model the resulting dynamo by a traditional $\alpha \omega$ formulation? Finally, what is the impact of the layer of shear in organizing the magnetic field: how does a purely convective dynamo differ from one with convection over a layer of shear? We set up the system in $\S 2$, explore these and other issues in $\S \S 3-4$, and give concluding discussion in $\S 5$.

\section{Governing equations}

Our starting point is the system of equations for Boussinesq convection including a magnetic field and Lorentz force feedback, governed by

$$
\begin{aligned}
\rho_{0}\left(\partial_{t} \boldsymbol{U}+\boldsymbol{U} \cdot \nabla \boldsymbol{U}+2 \boldsymbol{\Omega} \times \boldsymbol{U}\right) & =-\nabla \Pi+\rho \boldsymbol{g}+\mu^{-1}(\nabla \times \boldsymbol{B}) \times \boldsymbol{B}+\rho_{0} v \nabla^{2} \boldsymbol{U}, \\
\partial_{t} \boldsymbol{B} & =\nabla \times(\boldsymbol{U} \times \boldsymbol{B})+\eta \nabla^{2} \boldsymbol{B}, \\
\partial_{t} T+\boldsymbol{U} \cdot \nabla T & =\kappa \nabla^{2} T, \\
\nabla \cdot \boldsymbol{U} & =0, \quad \nabla \cdot \boldsymbol{B}=0,
\end{aligned}
$$

and $\rho=\rho_{0}\left(1-\alpha\left(T-T_{0}\right)\right)$.

We adopt a plane-layer geometry, having in mind a local approximation at colatitude $\vartheta$ to a rotating spherical shell of fluid, with $z$ pointing vertically upwards, $x$ east and $y$ north, as indicated in figure 1 . We therefore take the acceleration due to gravity,

$$
\boldsymbol{g}=-g \boldsymbol{e}_{z}
$$

and rotation vector

$$
\boldsymbol{\Omega}=\Omega \hat{\boldsymbol{\Omega}}=\Omega\left(\sin \vartheta \boldsymbol{e}_{y}+\cos \vartheta \boldsymbol{e}_{z}\right)
$$

The plane layer is defined by $0 \leqslant z \leqslant h$ and we take it to be periodic in $x$ and $y$ with periods $h L_{x} \equiv 2 \pi h / k_{x}$ and $h L_{y} \equiv 2 \pi h / k_{y}$, respectively. 
We impose perfectly conducting boundary conditions

$$
B_{z}=0, \quad \partial_{z} B_{x}=\partial_{z} B_{y}=0 \quad(z=0, h),
$$

at the top and bottom of the layer. We allow a temperature contrast to drive convection, and use no-slip boundary conditions for the flow, with

$$
\begin{array}{cl}
\boldsymbol{U}=U_{0} \boldsymbol{e}_{x}, & T=T_{0}+\Delta T \quad(z=0), \\
\boldsymbol{U}=0, & T=T_{0} \quad(z=h) .
\end{array}
$$

The bottom boundary has a velocity $U_{0}$ in the $x$-direction, which will drive a shear flow across the plane layer, as in paper I.

This system is non-dimensionalized using the scale $h$ and the thermal time scale $h^{2} / \kappa$ by setting

$$
\begin{gathered}
\boldsymbol{x}=h \boldsymbol{x}^{\prime}, \quad t=\frac{h^{2}}{\kappa} t^{\prime}, \quad \boldsymbol{U}=\frac{\kappa}{h} \boldsymbol{U}^{\prime}, \\
\theta=\frac{\nu \kappa}{\alpha g h^{3}} \theta^{\prime}, \quad \Pi=\frac{\rho_{0} \kappa^{2}}{h^{2}} \Pi^{\prime}, \quad \boldsymbol{B}=\frac{\left(\rho_{0} \mu\right)^{1 / 2} \kappa}{h} \boldsymbol{B}^{\prime},
\end{gathered}
$$

where $\theta$ is the deviation from the linear, conductive profile satisfying the boundary conditions. After dropping the dashes, the system becomes

$$
\begin{aligned}
\partial_{t} \boldsymbol{U}+\boldsymbol{U} \cdot \nabla \boldsymbol{U}+\tau P \widehat{\boldsymbol{\Omega}} \times \boldsymbol{U} & =-\nabla \Pi+P \theta \boldsymbol{e}_{z}+(\nabla \times \boldsymbol{B}) \times \boldsymbol{B}+P \nabla^{2} \boldsymbol{U}, \\
\partial_{t} \theta+\boldsymbol{U} \cdot \nabla \theta & =R a U_{z}+\nabla^{2} \theta, \\
\partial_{t} \boldsymbol{B} & =\nabla \times(\boldsymbol{U} \times \boldsymbol{B})+q^{-1} \nabla^{2} \boldsymbol{B}, \\
\nabla \cdot \boldsymbol{U} & =0, \quad \nabla \cdot \boldsymbol{B}=0,
\end{aligned}
$$

with boundary conditions for temperature deviation and magnetic field

$$
\theta=B_{z}=\partial_{z} B_{x}=\partial_{z} B_{y}=0 \quad(z=0,1)
$$

and for the flow,

$$
\boldsymbol{U}=\operatorname{RePe}_{x} \quad(z=0), \quad \boldsymbol{U}=0 \quad(z=1) .
$$

The dimensionless parameters introduced are

$$
R a=\frac{\alpha g h^{3} \Delta T}{\nu \kappa}, \quad R e=\frac{U_{0} h}{v}, \quad \tau=\frac{2 \Omega h^{2}}{v}, \quad P=\frac{\nu}{\kappa}, \quad q=\frac{\kappa}{\eta},
$$

that is, the Rayleigh number, Reynolds number, the square root of the Taylor number, the Prandtl number and the Roberts number. Our plane-layer magnetohydrodynamical system is completely specified by the parameter set

$$
\left\{R a, R e, \tau, P, q, \vartheta, k_{x}, k_{y}\right\} \text {. }
$$

Note that the Reynolds number $R e$ is based on the imposed velocity $U_{0}$ at the base of the layer, and not on a measure of the flow velocity actually realized, which could be different (for example if the flow were driven only by convection). We take $\tau \geqslant 0$ and $0 \leqslant \vartheta \leqslant \pi / 2$ (northern hemisphere), but allow the parameter $R e$ to take either sign, corresponding to the sign of $U_{0}$.

The system permits a solution depending only on the vertical coordinate $z$, in which $\boldsymbol{B}=0, \theta=0$ and the flow profile takes an Ekman-Couette form,

$$
\boldsymbol{U}_{E k}(z)=\operatorname{ReP} \boldsymbol{\Lambda}(z)=\operatorname{ReP}\left(\Lambda_{1}(z) \boldsymbol{e}_{x}+\Lambda_{2}(z) \boldsymbol{e}_{y}\right)
$$


where the $\Lambda_{i}(z)$ satisfy

$$
-2 \mu^{2} \Lambda_{2}=\Lambda_{1}^{\prime \prime}, \quad 2 \mu^{2} \Lambda_{1}=\Lambda_{2}^{\prime \prime}, \quad \mu \equiv \sqrt{\frac{1}{2} \tau \cos \vartheta},
$$

with $\Lambda_{1}(0)=1$ and $\Lambda_{2}(0)=\Lambda_{1}(1)=\Lambda_{2}(1)=0$. The full solution is given in equation (29) of paper I; we simply note that in the limit of large $\tau$ (for $\vartheta \neq \pi / 2$ ), the flow becomes an Ekman layer localized at the bottom boundary,

$$
\Lambda_{1}(z) \simeq \mathrm{e}^{-\mu z} \cos \mu z, \quad \Lambda_{2}(z) \simeq-\mathrm{e}^{-\mu z} \sin \mu z \quad(\mu \gg 1),
$$

(there being no horizontal pressure gradient imposed in the system). Note that there is a net flux in the $-y$-direction in $\boldsymbol{U}_{E k}$ for $\operatorname{ReP}>0$, with maximum $y$-velocity

$$
\max _{z} U_{E k, y}=-2^{-1 / 2} \mathrm{e}^{-\pi / 4} R e P \simeq-0.3224 R e P \quad \text { at } \quad z_{\max }=\pi / 4 \mu,
$$

and $z_{\max }$ gives a convenient measure of the Ekman layer thickness. Sometimes it is useful to subtract the Ekman-layer flow, giving

$$
\boldsymbol{U}=\operatorname{ReP\Lambda }(z)+\boldsymbol{u} .
$$

This system allows a rich family of instabilities: convective instability is possible for $R a>0$, Taylor-Couette $(\vartheta=\pi / 2)$ for $R e>0$, and Ekman $(\vartheta \neq \pi / 2)$ for $R e \neq 0$, as discussed in Ponty et al. (2003). As a rule of thumb, configurations with $R e>0$ are generally less stable than those with $R e<0$. In this paper, we will only consider configurations with positive values of $R e$.

The shear flow (2.20) on its own cannot support dynamo action, and so it is necessary to follow a hydrodynamic or Ekman instability to a saturated state, and then introduce a magnetic field. The kinematic evolution of such fields was studied in a two-dimensional setting in paper I. To follow their nonlinear evolution and saturation, a three-dimensional finite-difference code was written. This time steps the system (2.12)-(2.15), written in terms of $\boldsymbol{u}$ from (2.24) with homogeneous boundary conditions applied to $\theta, \boldsymbol{B}$ and $\boldsymbol{u}$ (see (2.16), (2.17)); further details are given in Appendix A.

\section{Dynamo action in convection with shear}

\subsection{Parameters and kinematic evolution}

In undertaking three-dimensional simulations, the scope to explore parameter space is limited. The main run we study in this paper has the parameter values

$$
\begin{gathered}
R a=7500 \simeq 2 R a_{c}, \quad R e=30, \quad \tau=200, \\
P=1, \quad q=50, \quad \vartheta=67.5^{\circ}, \quad k_{x}=4.30, \quad k_{y}=1.0,
\end{gathered}
$$

so that the box has dimensions of $L_{x} \times L_{y} \times 1 \simeq 1.46 \times 6.28 \times 1$. These were chosen to build on earlier work in paper I: the $x$-dimension gives the preferred scale of convective rolls at onset, while the $y$-dimension is sufficiently large to allow the field to develop structure on a range of scales. Our dynamical run is closely related to a purely kinematic run shown in figure $7 \dagger$ of paper I, for which the parameters are similar. The only important difference is that in paper I the Rayleigh number $R a \simeq 1.1 R a_{c}$ is close to critical, whereas ours is twice critical. 
(a)

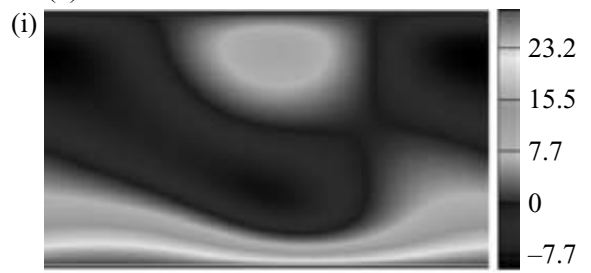

(ii)

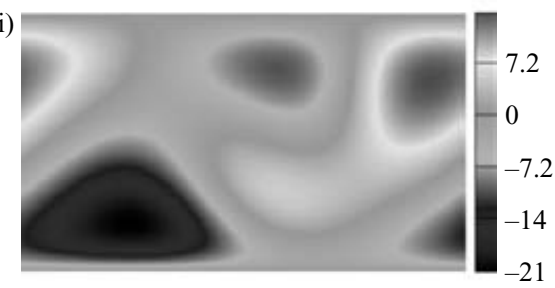

(iii)

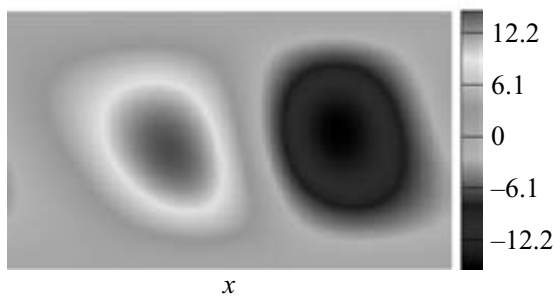

(b)
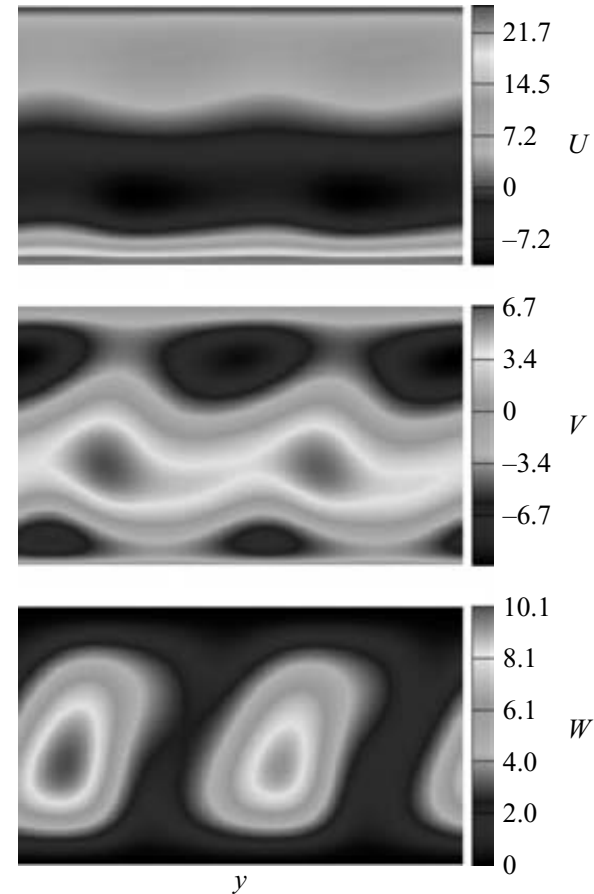

Figure 2. Convective flow in the kinematic regime, with parameter values (3.1), (3.2). The flow components $(U, V, W)$ of $\boldsymbol{U}$ in $(a)$ the $(x, z)$-plane and $(b)$ the $(y, z)$-plane.

In the absence of convection and magnetic field, the values of $R e$ and $\tau$ lead to an Ekman layer localized near to the base of the plane layer, with thickness $z_{\max } \simeq 0.127$ in (2.23). The effect of then raising the Rayleigh number just above critical, as in paper I, is to generate convective rolls which tend to lie above the Ekman layer (being suppressed within the layer by the strong shear). The orientation of the convective rolls is dominated by the rotation of the system and their axes are approximately aligned with the horizontal component of $\boldsymbol{\Omega}$, that is, with the $y$-direction. The effect of the Ekman layer is to change the orientation of the rolls at onset in an infinite plane layer, from exact alignment, by an angle $\epsilon \simeq 2.33^{\circ}$. The effect is small because the localized Ekman layer has little interaction with the convection at these parameter values (see paper I and references therein). In our finite periodic plane-layer geometry, the set of possible wavevectors is limited and so at onset, the roll axes are exactly aligned with the $y$-direction, the resulting flow being shown schematically in figure $1(c)$.

In our run, the Rayleigh number is increased to $R a \simeq 2 R a_{c}$, which has two effects. First, the convection becomes more vigorous, although not so strong as to disrupt the Ekman layer. Secondly, the flow bifurcates at $R a \simeq 1.7 R a_{c}$, and by $R a \simeq 2 R a_{c}$ has modest three-dimensionality, although it remains steady in a moving frame. The flow takes the form of convective rolls with some variation in the $y$-direction, as depicted in figure 2. Figure 2(a) shows the flow components on a slice of constant $y$ and figure $2(b)$ shows a slice of constant $x$. Clearly visible in $U$ (figure $2 a(i)$ ) is the Ekman layer at the base of the layer; the $W$ component in the $(x, z)$-plane (figure $2 a($ iii)) indicates the presence of two convective rolls, while figure $2(b)$ shows the three-dimensionality of the flow. 
(a)

(i)

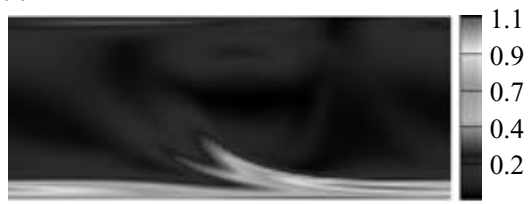

(ii)

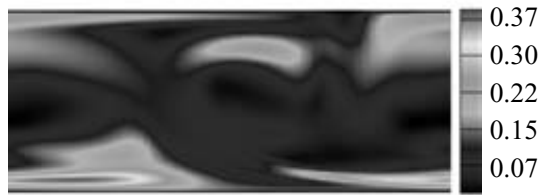

(iii)

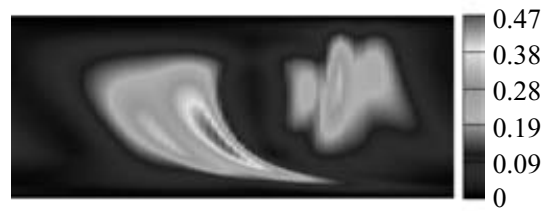

(iv)

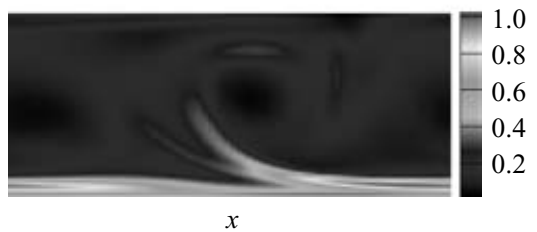

(b)
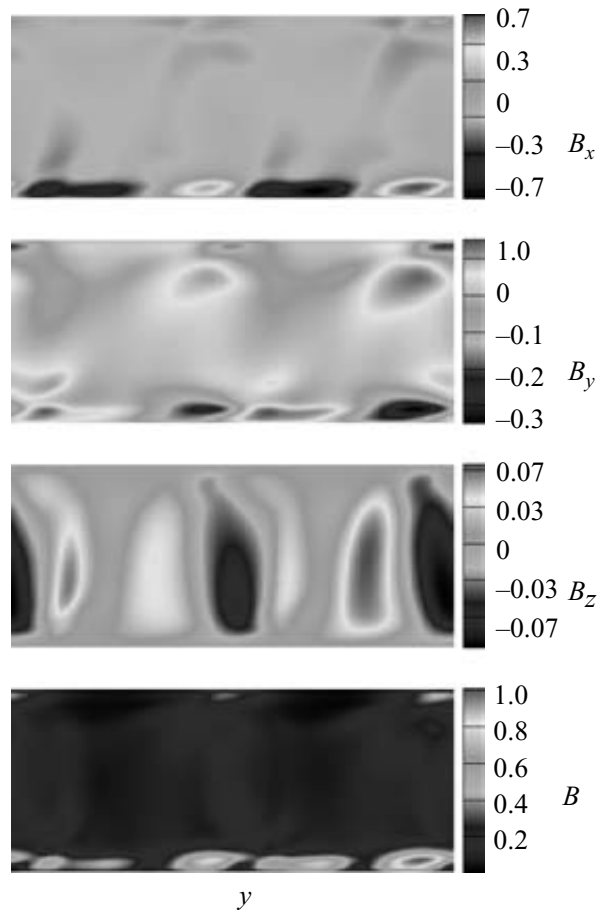

FIGURE 3. Magnetic field in the kinematic regime, with parameter values (3.1), (3.2). The field components $B_{x}, B_{y}, B_{z}$, and $B=|\boldsymbol{B}|$ in $(a)$ the $(x, z)$-plane and $(b)$ the $(y, z)$-plane; see (3.3) and (3.4).

The magnetic field in the kinematic regime is shown in figure 3. Different quantities are plotted to indicate different aspects of the magnetic field structure. Figure 3(a) shows

$$
\sqrt{\left\langle B_{x}^{2}\right\rangle_{y}}, \quad \sqrt{\left\langle B_{y}^{2}\right\rangle_{y}}, \quad \sqrt{\left\langle B_{z}^{2}\right\rangle_{y}}, \quad \sqrt{\left\langle|\boldsymbol{B}|^{2}\right\rangle_{y}},
$$

i.e. the field intensity, averaged over $y$. Figure $3(b)$ shows

$$
\left\langle B_{x}\right\rangle_{x}, \quad\left\langle B_{y}\right\rangle_{x}, \quad\left\langle B_{z}\right\rangle_{x}, \quad \sqrt{\left\langle|\boldsymbol{B}|^{2}\right\rangle_{x}},
$$

and so gives an indication of the sign of the field (except figure $3 b$ (iv)).

In figures 3(a)(iv) and 3(b)(iv) we see that most of the magnetic energy is in the form of structures that are extended in the $x$-direction, and the field is predominantly $x$-directed. The structures are flattened in the $y$-direction as seen in figure $3(b)(\mathrm{i})$, and so form what we will refer to as sheets of field. This figure indicates that there are essentially 4 sheets, 2 with field pointing in the $-x$-direction and 2 in the $+x$-direction. This corresponds to a dominant $n=2$ mode, where it is convenient to decompose the field as

$$
\boldsymbol{B}(x, y, z, t)=\sum_{n} \hat{\boldsymbol{B}}_{n}(x, z, t) \exp \left(\mathrm{in} k_{y} y\right) .
$$

In paper I, these modes were decoupled as the flow was $y$-independent, but for the present parameter values the modes are coupled because of three-dimensionality in the flow field. Note that in paper I, insulating boundary conditions were employed; we use perfectly conducting boundary conditions which have more of a trapping effect 


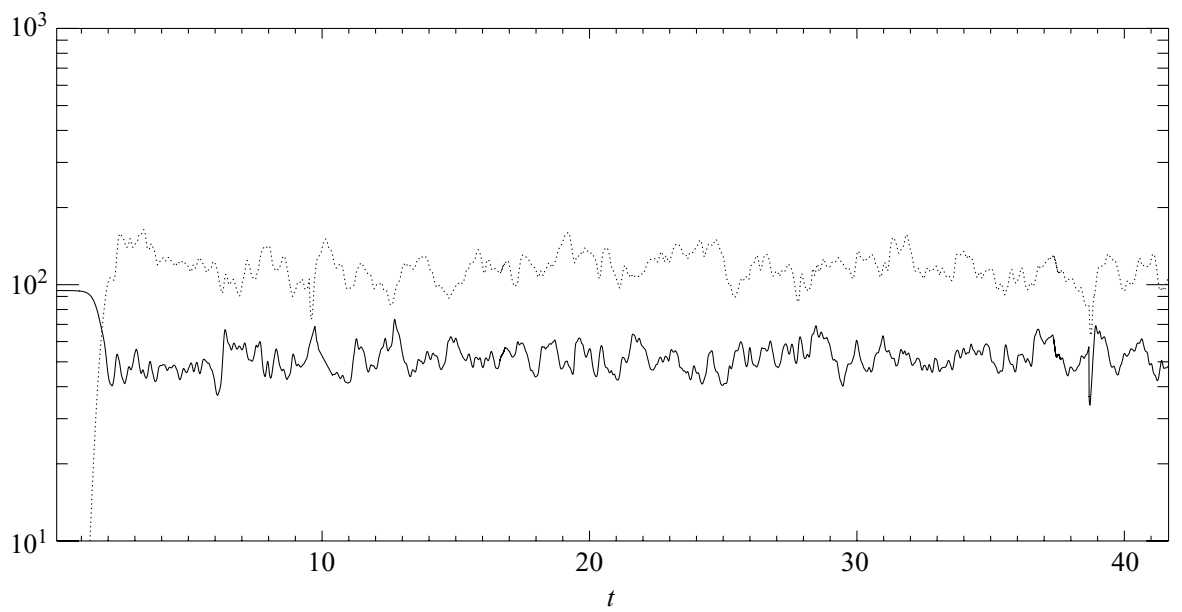

FIGURE 4. The kinetic energy $E_{K}$ (solid) and the magnetic energy $E_{M}$ (dotted) plotted on a logarithmic scale as functions of time.

of field at the boundary. Tests indicate that this makes little difference to magnetic field structure and kinematic growth rate in the presence of strong shear.

The magnetic field moves as a wave propagating in the $-y$-direction, an aspect we will return to later. Although the field lies predominantly within the shear layer (see also figure 8 below), the convection above it is important in drawing out tongues of field, which are then folded back into the shear flow. This is particularly seen in the $B_{z}$ field, which suggests the dynamo could be classified as of $\alpha \omega$ type, with the convection providing an $\alpha$ effect; we will discuss this further below.

\subsection{Energies and transfers}

Our main run starts with a seed magnetic field and the equilibrated convective flow (figure 2), and follows the magnetic field through kinematic growth (figure 3) to saturation. The key issue is to find suitable diagnostics to understand the wealth of data potentially available. Two diagnostics are the total kinetic and magnetic energies,

$$
E_{K}=\frac{1}{2}\left\langle|\boldsymbol{U}|^{2}\right\rangle, \quad E_{M}=\frac{1}{2}\left\langle|\boldsymbol{B}|^{2}\right\rangle,
$$

where $\langle\cdot\rangle$ denotes an average over the periodic box. Figure 4 shows the energies plotted as a function of time.

In the kinematic regime, $E_{K} \simeq 95$ and the magnetic field grows as $E_{M} \propto \mathrm{e}^{2 \sigma t}$ with growth rate $\sigma \simeq 3.05$. In our system, the magnetic Reynolds number $R m$ is a diagnostic, which depends on the flow that is realized. Using the root-mean-square velocity $U$ that is measured in the simulation, we define $R m$ in our non-dimensionalization by

$$
R m=U q, \quad U \equiv \sqrt{2 E_{K}} .
$$

Kinematically $R m \simeq 690$, and the field has correspondingly fine-scale structure, as seen in figure 3.

The field saturates at $t \simeq 3$, leading to a state with complicated time-dependence and relatively strong magnetic fields. Despite the large value of $\mathrm{Rm}$, the magnetic energy is about twice that of the kinetic energy, with

$$
\left\langle E_{K}\right\rangle_{t} \simeq 50, \quad\left\langle E_{M}\right\rangle_{t} \simeq 120,
$$

and $R m$ reduced to $R m \simeq 510$. 
(a) 10

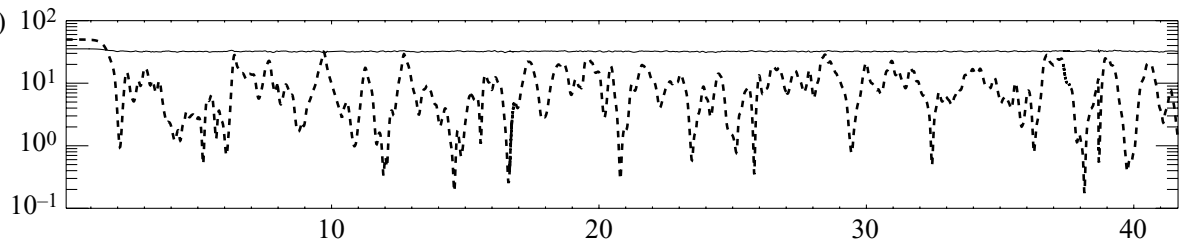

(b)

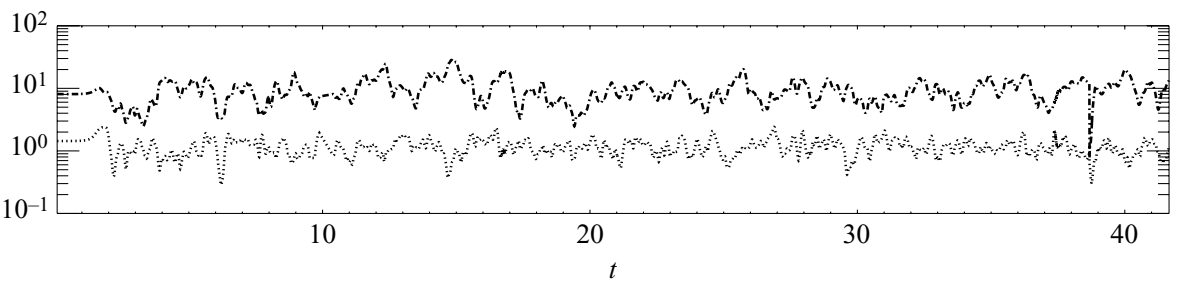

FIGURE 5. The kinetic energy components $(a) E_{K 1}$ (solid), $E_{K 2 x}$ (dash), (b) $E_{K 2 y}$ (dot) and $E_{K 3}$ (dash-dot).

To understand the nature of the saturation in more detail, we decompose the velocity field into four components with distinct dependencies on the coordinates, and corresponding energies,

$$
\begin{aligned}
\boldsymbol{U} & =\boldsymbol{U}_{1}(z)+\boldsymbol{U}_{2 x}(x, z)+\boldsymbol{U}_{2 y}(y, z)+\boldsymbol{U}_{3}(x, y, z), \\
E_{K} & =E_{K 1}+E_{K 2 x}+E_{K 2 y}+E_{K 3},
\end{aligned}
$$

with

$$
\boldsymbol{U}_{1}=\langle\boldsymbol{U}\rangle_{x, y}, \quad \boldsymbol{U}_{2 x}=\left\langle\boldsymbol{U}-\boldsymbol{U}_{1}\right\rangle_{y}, \quad \boldsymbol{U}_{2 y}=\left\langle\boldsymbol{U}-\boldsymbol{U}_{1}\right\rangle_{x} .
$$

This breaks the flow into a shear component $\boldsymbol{U}_{1}$, a component $\boldsymbol{U}_{2 x}$ independent of $y$, a component $\boldsymbol{U}_{2 y}$ independent of $x$, and the remainder, a three-dimensional component $\boldsymbol{U}_{3}$. If the flow were written in terms of Fourier modes $\exp \left(\mathrm{i} m k_{x} x+\mathrm{i} n k_{y} y\right)$, these four components would correspond to: the $(0,0)$ mode, the modes $(m, 0)$ with $m \neq 0$, the modes $(0, n)$ with $n \neq 0$, and the modes $(m, n)$ with both $m \neq 0$ and $n \neq 0$, respectively.

Figure 5 shows these kinetic energy components as functions of time. Focusing first on the kinematic regime $t<1.5$, the energy in the shear flow $E_{K 1} \simeq 36$ (solid), dominated by the Ekman layer, is broadly similar to that in the $y$-independent component $E_{K 2 x} \simeq 50$ (dash), which is dominated by the convective cells. The modest three-dimensionality of the flow is indicated by the presence of the components $E_{K 2 y} \simeq 1.4$ and $E_{K 3} \simeq 8$.

In the dynamical regime $t>3$, the most significant feature is that the energy in the shear flow $E_{K 1}$ (solid) is relatively unchanged. The magnetic field saturates while having little effect on the underlying Ekman-layer flow. Measurements of the discrepancy from the Ekman profile, measured by $\left|\boldsymbol{U}_{1}-\boldsymbol{U}_{E k}\right|^{2} / 2$ (not plotted here) show small fluctuations with time. In effect, $\boldsymbol{U}_{1} \simeq \boldsymbol{U}_{E k}$ even in the dynamical regime. The principal effect of the Lorentz force is to suppress the convection above the Ekman layer. The energy component $E_{K 2 x}$ (dash) in figure 5 shows a marked reduction from kinematic values, and then a bursty behaviour, varying from around 1 up to values as high as 25 .

Information about how the magnetic field is sustained within the dynamical regime may be obtained from the energy equation for $\boldsymbol{B}$,

$$
\partial_{t} E_{M}=\left\langle B_{i} B_{j} e_{i j}\right\rangle-q^{-1}\left\langle|\nabla \times \boldsymbol{B}|^{2}\right\rangle .
$$



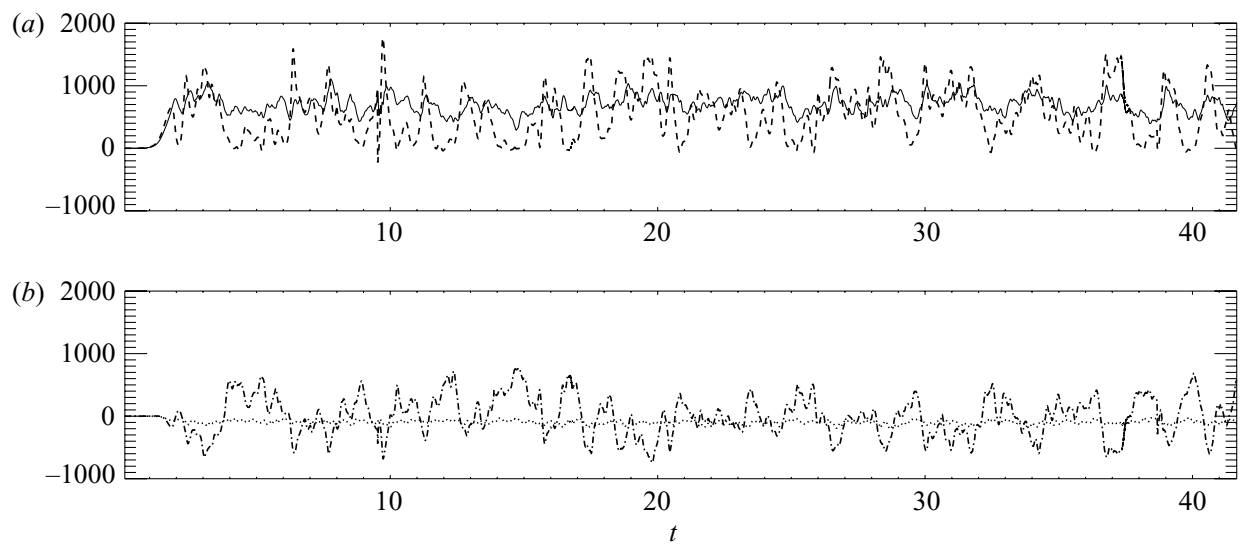

FIGURE 6. The transfer terms (a) $T_{1}$ (solid), $T_{2 x}$ (dash), (b) $T_{2 y}$ (dot) and $T_{3}$ (dash-dot).

The input of magnetic energy by stretching in the fluid flow is written in terms of the rate of strain tensor $e_{i j}=\left(\partial_{i} U_{j}+\partial_{j} U_{i}\right) / 2$. The corresponding energy equation for $\boldsymbol{U}$ is

$$
\partial_{t} E_{K}=-\left\langle B_{i} B_{j} e_{i j}\right\rangle+P\left\langle U_{z} \theta\right\rangle-P\left\langle|\nabla U|^{2}\right\rangle-\operatorname{ReP}\left\langle\left.\partial_{z} U_{x}\right|_{z=0}\right\rangle_{x, y},
$$

where the last term gives the input of kinetic energy through the motion of the lower boundary.

We focus on the key transfer term $T=\left\langle B_{i} B_{j} e_{i j}\right\rangle$, which may also be decomposed into contributions $T_{1}, T_{2 x}, T_{2 y}$ and $T_{3}$, from the distinct flow components $\boldsymbol{U}_{1}, \boldsymbol{U}_{2 x}$, $\boldsymbol{U}_{2 y}$ and $\boldsymbol{U}_{3}$. Figure 6 shows these individual transfer terms as functions of time. It may be seen that there is a consistent transfer $T_{1}$ (solid) of energy into the magnetic field from the shear flow $\boldsymbol{U}_{1}$, although this fluctuates much more than does the shear flow itself (see figure 5). The convective flow component $\boldsymbol{U}_{2 x}$ also gives a net input of energy $T_{2 x}$ (dash) into the field, correlated with the strength of the flow itself (see $E_{2 x}$ in figure 5), and so showing large fluctuations with time. On the other hand, there is a net loss of magnetic energy into the weak flow $\boldsymbol{U}_{2 y}$, and no persistent sign of transfer for the final three-dimensional component $\boldsymbol{U}_{3}$.

\subsection{Magnetic field and flow structure}

A snap-shot of the magnetic field in the saturated state is shown in figure 7. This may be compared with the kinematic field in figure 3. We observe that the field remains largely localized in the Ekman shear layer (whose thickness remains $z_{\max } \simeq 0.13$ dynamically), but instead of taking the form of sheets, appears to show structures extended only in the $x$-direction, which we refer to as tubes of field (see, for example, figures $7 a(\mathrm{i})$ and $7 b(\mathrm{i})$ and bear in mind the $2 \pi \times 1$ dimensions in the $(y, z)$-plane).

To quantify the spatial localization of magnetic field, figure 8 shows the magnetic energy $E_{M}(z)$ as a function of $z$,

$$
E_{M}(z)=\frac{1}{2}\left\langle|\boldsymbol{B}|^{2}\right\rangle_{x, y}
$$

that is, averaged over $x$ and $y$ only. In figure 8(a) the profile is shown for the early, kinematic regime, while in figure $8(b)$ a series of curves shows the profile at a number of later times. We observe that, in each case, the field is localized in the Ekman layer, but dynamically it broadens in scale. There is some effect of the perfectly conducting boundary conditions in trapping magnetic field, at the top boundary, but the strongest fields are localized away from the bottom boundary, in the region of maximum shear. 
(a)

(i)

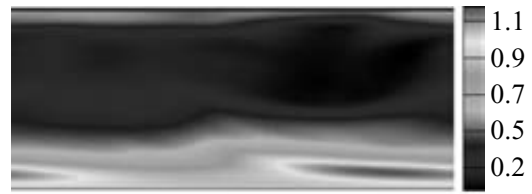

(ii)

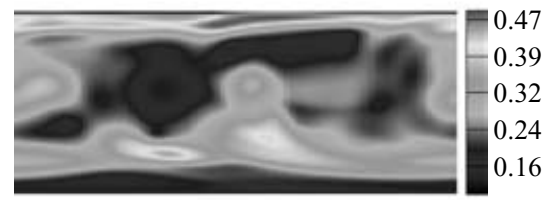

(iii)

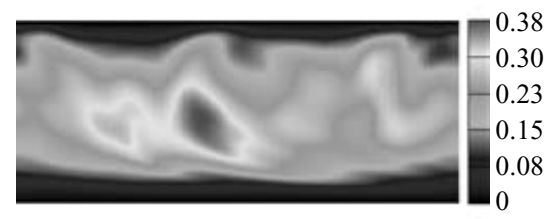

(iv)

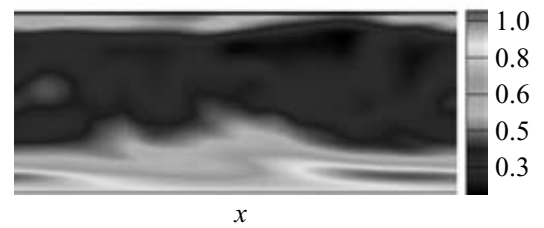

(b)
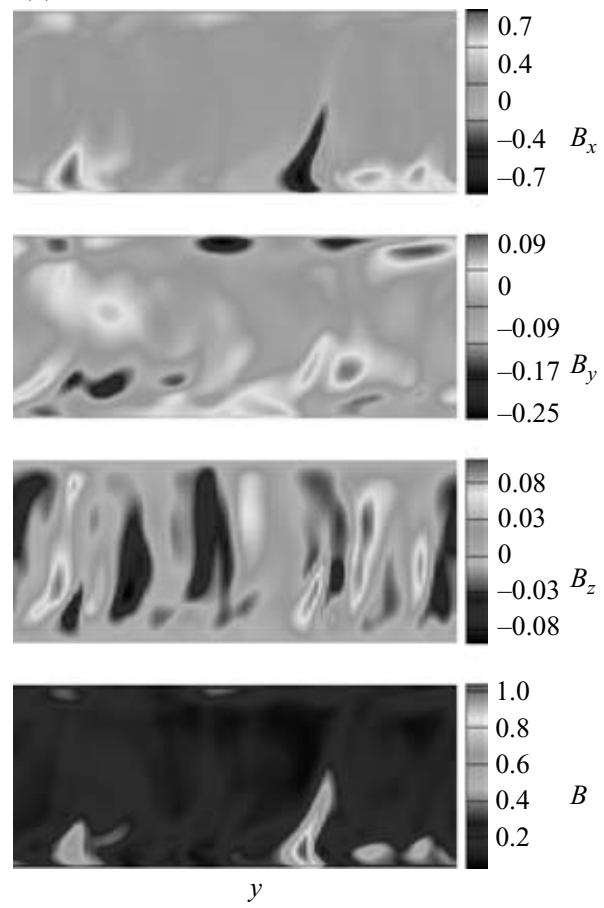

FIgURE 7. Magnetic field for parameter values (3.1), (3.2), in the dynamical regime, at $t=24$. The plots are as in figure 3 .
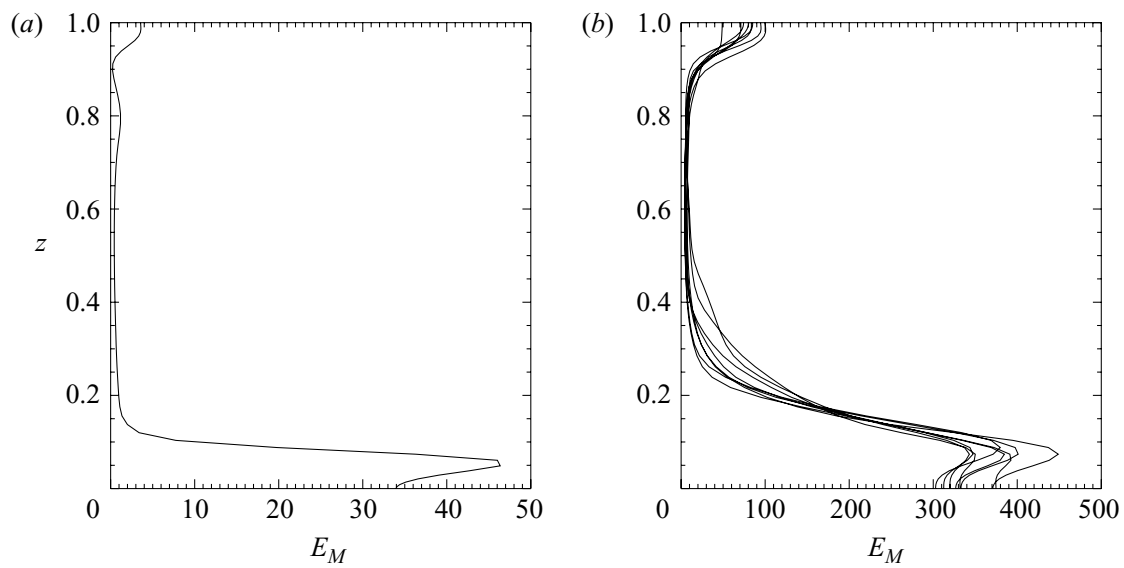

Figure 8. Magnetic field profiles $(a)$ in the kinematic regime, $(b)$ at a number of times in the dynamical regime. Plotted is $E_{M}(z)$ (horizontal axis) against $z$ (vertical axis).

Figure 9 shows a similar comparison of the kinetic energy $E(z)-E_{1}(z)$ (taking out the dominating shear component) for kinematic and dynamical regimes. The flow shows considerable variation in time, but is generally suppressed dynamically. We do not plot helicity here: the helicity is dominated by that in the Ekman flow $\boldsymbol{U}_{1}$. When this is excluded, the remaining flow has a helicity profile that we measure very approximately as

$$
H(z)-H_{1}(z)=\left\langle\left(\boldsymbol{U}-\boldsymbol{U}_{1}\right) \cdot \nabla \times\left(\boldsymbol{U}-\boldsymbol{U}_{1}\right)\right\rangle_{x, y} \simeq h_{0} \sin 2 \pi z, \quad h_{0} \simeq 550,
$$




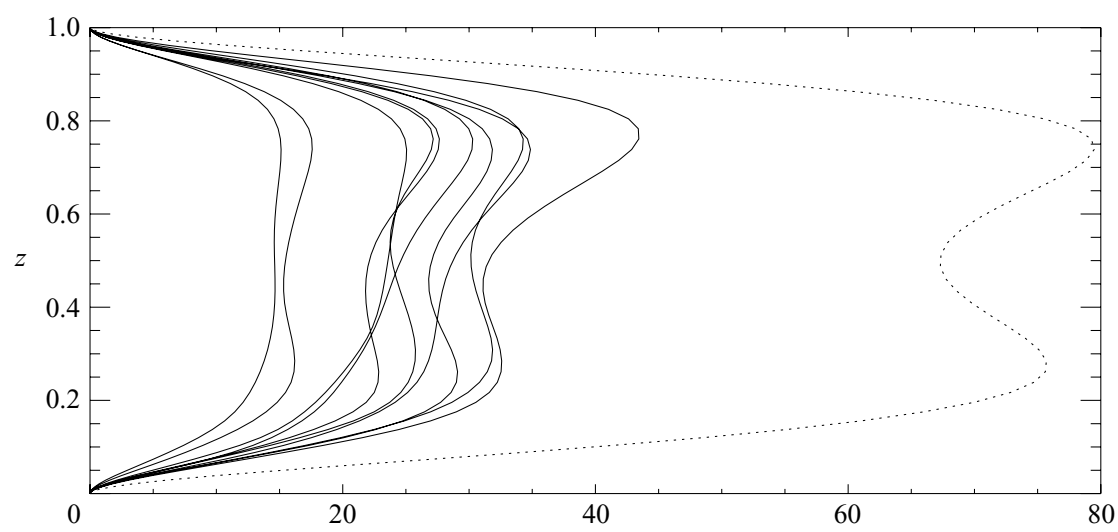

FIGURE 9. Fluid flow energy profiles in the kinematic regime (dotted), and at a number of times in the dynamical regime (solid). Plotted is $E_{K}(z)-E_{K 1}(z)$ (horizontal axis) against $z$ (vertical axis).

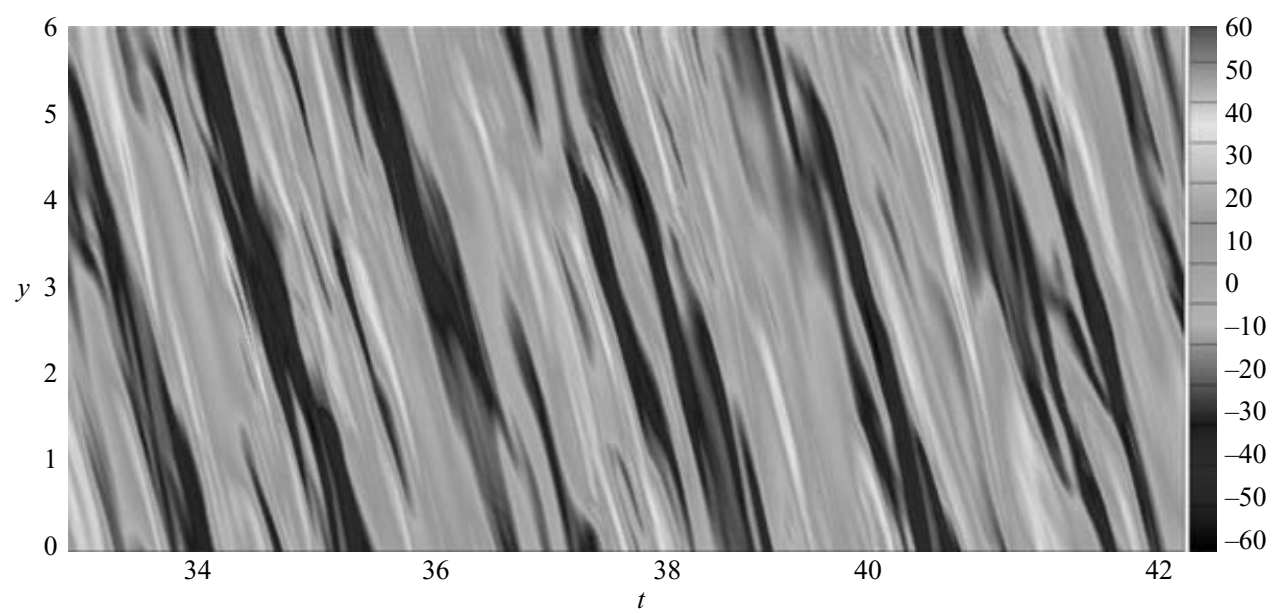

FIGURE 10. Butterfly diagram for the magnetic field diagnostic (3.16), plotted in the $(t, y)$-plane.

in kinematic regimes. This is antisymmetric about the mid-point $z=1 / 2$ as we would expect for rotating convection (e.g. Childress \& Soward 1972), while dynamically this helicity is suppressed.

The tubes seen in figure 7 translate in the $-y$-direction, the direction of the $y$ component of the Ekman flow (see (2.23)) but also evolve in time. As they are extended in the $x$-direction and confined to a narrow band of $z$-values, it is useful to plot an average over $x$ and a slice at $z=z_{\max }$,

$$
\hat{B}_{x}(y, t)=\left\langle B_{x}\left(x, y, z_{\max }, t\right)\right\rangle_{x}
$$

as a function of $t$ and $y$. This is done in figure 10 as a plot in the style of a butterfly diagram'. Each tube is revealed as a streak that crosses the plane, with life-times comparable to the transit time of the wave. The diagram also reveals a larger-scale coherence in the field, which shows groupings of tubes of similar sign, corresponding 


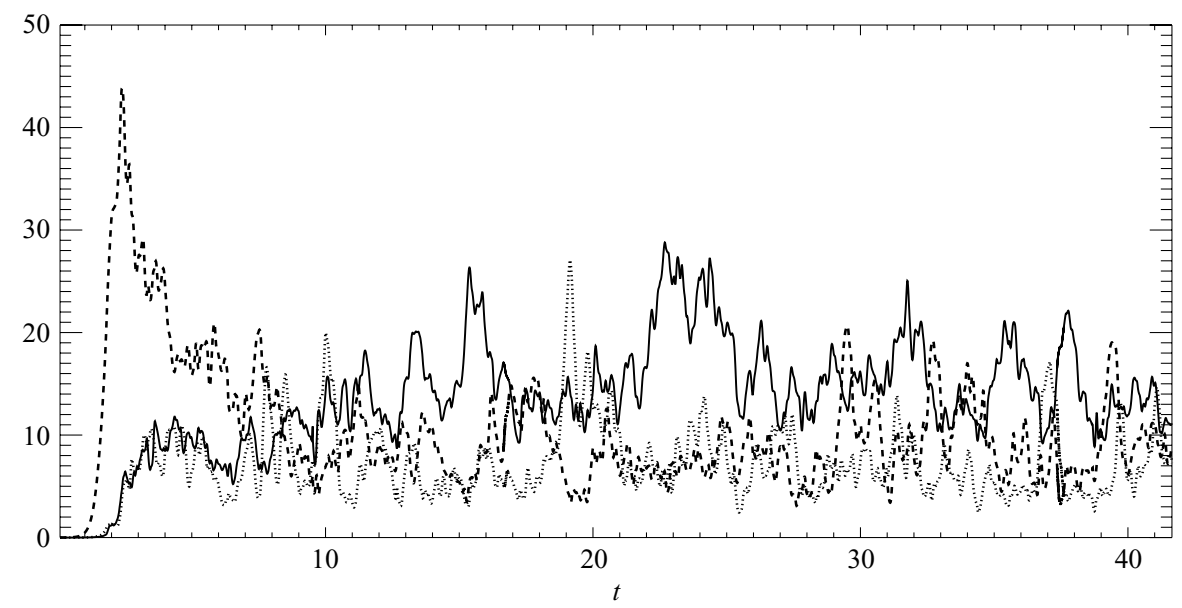

FiguRE 11. Magnetic energies $E_{M(n)}$ as a function of time with with $n=1$ (solid), $n=2$ (dash), $n=3$ (dot).

to mode $n=1$ in the $y$-direction (with modes defined by $\mathrm{e}^{\mathrm{i} n y}$ as $y$ varies from 0 to $2 \pi$ here).

The loss of coherence of the magnetic field on smaller scales, sheets breaking into tubes, is in accord with the results shown in figures 5 and 6 . While the shear flow itself remains largely time-independent, the transfer of energy $T_{1}$ by stretching of magnetic field in the shear becomes more intermittent, presumably as tubes of field are carried into and out of the shear by the up-down convective motions.

To quantify the larger-scale modulation apparent in figure 10 , we consider the energies $E_{M(n)}$ in each of the magnetic modes in (3.5). Here, we set

$$
E_{M(0)}=\frac{1}{2}\left\langle\left|\hat{\boldsymbol{B}}_{0}(x, z)\right|^{2}\right\rangle, \quad E_{M(n)}=\left\langle\left|\hat{\boldsymbol{B}}_{n}(x, z)\right|^{2}\right\rangle \quad(n \geqslant 1) .
$$

so that

$$
E_{M}=\sum_{n=0}^{\infty} E_{M(n)}
$$

Figure 11 gives these energies as a function of time. We see that after an initial phase in which the mode $n=2$ is dominant, including the kinematic phase, mode 1 takes over and the field adopts the largest scale available to it in the $y$-direction. Note, however, that there are periods when other modes rise to similar levels to mode 1 , and then subside, e.g. $t \simeq 20$. In fact, all modes plotted have comparable energies.

Although the dominance of mode 1 is unclear in figure 11 there is some evidence that it is in fact controlling the functioning of the dynamo. In fluid flows dominated by shear, it is natural to set up an $\alpha \omega$ model, in which the convective fluid motions are parameterized by a transport coefficient $\alpha$. Appendix B outlines a basic model of this form. The key aspect we note is that such a model predicts dynamo waves. In these waves, the mean $B_{x}$ field is linked to the $B_{z}$ field with a fixed phase shift. Although it is difficult to measure $\alpha$ at large magnetic Reynolds number and particularly in simulations such as ours because of the inhomogeneity of the system (i.e. lack of scale separation) and the time required to obtain sensible averages (e.g. Cattaneo, Hughes \& Thelen 2002), it is worthwhile seeing whether such a phase relation exists. We 
(a)

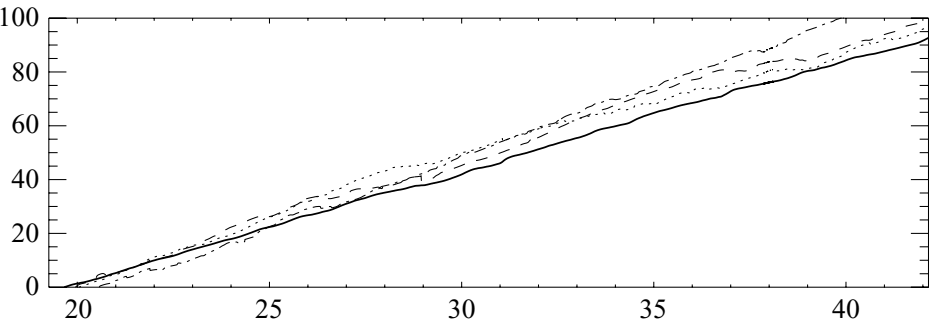

(b)

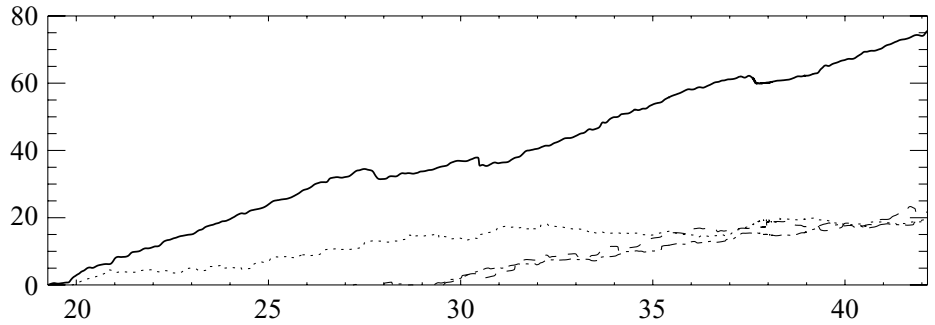

(c)

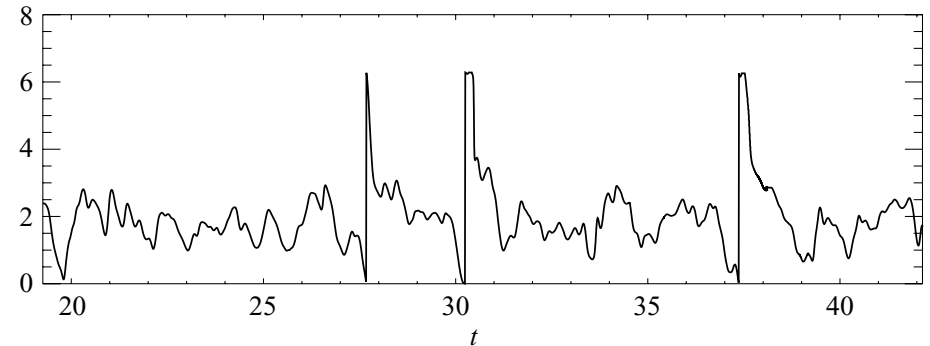

FigURE 12. Scaled phases $(a) \Phi_{n}(t) / k$ and $(b) \Psi_{n}(t) / k$ are plotted against time for $n=1$ (solid), $n=2$ (dash), $n=3$ (dot), $n=4$ (dash-dot). In $(c)$, the phase shift $\Psi_{n}(t)-\Phi_{n}(t)$ is plotted, modulo $2 \pi$, for $n=1$ only.

define phases $\Phi_{n}(t), \Psi_{n}(t)$ of the $x$ - and $z$-components of the magnetic modes (3.5),

$$
f_{n}(t) \mathrm{e}^{\mathrm{i} \Phi_{n}(t)}=\left\langle\boldsymbol{e}_{x} \cdot \hat{\boldsymbol{B}}_{n}\right\rangle_{x, z}, \quad g_{n}(t) \mathrm{e}^{\mathrm{i} \Psi_{n}(t)}=\left\langle\boldsymbol{e}_{z} \cdot \hat{\boldsymbol{B}}_{n}\right\rangle_{x, z} \cdot
$$

The instantaneous phase velocity of a mode is, using just the $x$-component,

$$
c_{n}=-\frac{1}{k} \frac{\mathrm{d} \Phi_{n}}{\mathrm{~d} t} \quad\left(k \equiv n k_{y}\right),
$$

though in a complex flow such as is realized in the nonlinear regimes we measure an average $c_{n}$ via the change in phase $\Delta \Phi_{n}$ over a period of time $\Delta t$.

Figure 12(a) shows the scaled phases $\Phi_{n}(t) / k$ of the $x$-component of field for $n=1-$ 4 and the latter part of the main run. There is clear approximate linear growth in all phases, corresponding to coherent structures involving many modes travelling with phase speed $c_{n} \simeq-4$ in the $y$-direction. Figure $12(b)$ shows the scaled phases $\Psi_{n}(t) / k$ for the $z$-component of field. Here, the only coherent behaviour is demonstrated by the $n=1$ mode, which shows linear growth with the same phase speed. For $n=1$ only, the phase shift $\Psi_{n}(t)-\Phi_{n}(t)$ between the $x$ and $z$ fields is plotted in figure $12(c)$. This shows some 'glitches' where it slips through $2 \pi$, but generally only varies in a moderate band of approximately 1-2.5. Plainly, the $x$ - and $z$-components of field are 


\begin{tabular}{rrrrrrr}
\hline$R e$ & $\tau$ & $q$ & $E_{K}$ & $E_{M}$ & $R m$ & $\left\langle U_{z} \theta\right\rangle$ \\
30 & 200 & 50 & 50 & 120 & 500 & 2700 \\
30 & 200 & 15 & 45 & 110 & 140 & 2000 \\
30 & 200 & 5 & 60 & 70 & 40 & 3300
\end{tabular}

TABLE 1. Time-averaged kinetic and magnetic energy at different $q$, together with magnetic Reynolds number and rate of working of the buoyancy force.

locked together in terms of phase for the $n=1$ mode only, with a phase shift similar to that predicted by the simple $\alpha \omega$ modelling given in Appendix B. Further comparison with the $\alpha \omega$ model is given in this appendix. Here, we note only that the phase velocity of the dynamo wave involves a competition between the Ekman pumping velocity in the $-y$-direction, and the velocity induced by $\alpha \omega$ dynamo processes, which drives field in the $+y$-direction.

In short, we conclude that in the full numerical simulation, magnetic-field generation is compatible with an $\alpha \omega$ dynamo wave, dominated by mode $n=1$, which modulates the smaller scale tubular structures adopted by the magnetic field.

\section{Dynamo action with varying parameters}

We have studied the saturation of a dynamo in which shear and convection played key roles, and in which the magnetic Reynolds number was large, allowing the field to adopt complex structure and a range of scales. It is of interest to vary all of the parameters $R e, R e, \tau, q$ from (3.1) and (3.2), to understand parameter dependence, but this is impractical. Instead we focus on our main run and consider reducing any one of these parameters to switch off one effect, holding the others constant, and see how the dynamo behaviour responds, looking for useful points of comparison.

\subsection{Runs with varying $q$}

We first consider reducing $q$, which will in turn tend to reduce the magnetic Reynolds number $R m$, although the latter, of course, is a diagnostic, depending on the actual flow realized. Table 1 gives results for runs at varying $q$. These runs were started using the field and flow from our main run at the time $t=24$ and the runs are rather shorter than the main run; we note that this does not allow the possibility of the field slowly evolving to a totally different regime. We see that there is little variation in the kinetic energy, which remains $E_{K} \simeq 50$. The magnetic energy increases from $E_{M} \simeq 70$ at $q=5$ to a plateau of around $E_{M} \simeq 120$ at $q=50$.

For $q=5$, the value of $R m \simeq 40$ is moderate, the dynamo is close to critical, and the convection vigorous, with more transfer of energy to the flow from the buoyancy force, as measured by $\left\langle U_{z} \theta\right\rangle$. Note that this transfer shows large fluctuations of $\pm 800-1000$ about these average values, as the magnetic field turns the convection off and on. At these low $q$ values, the field becomes broader in structure, as shown in figure 13 , but remains a clear $n=1$ dynamo wave, predominantly in the shear layer. The phase speed and phase shift remain relatively unchanged.

\subsection{Runs with varying $R e$}

Now we consider reducing the shear parameter $R e$ and so going from an $\alpha \omega$ dynamo to a purely convective dynamo. Table 2 shows results for the main run and two subsidiary runs beginning with weak initial magnetic fields. The most interesting 
(a)

(i)

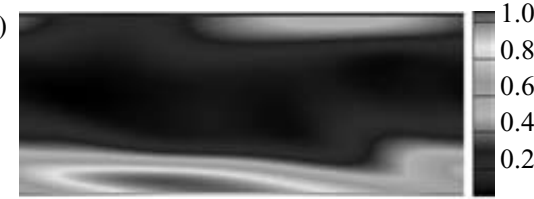

(ii)

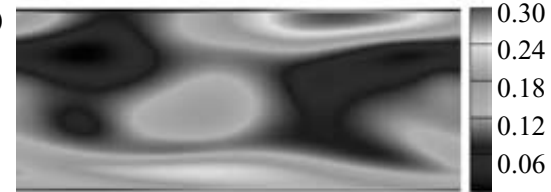

(iii)

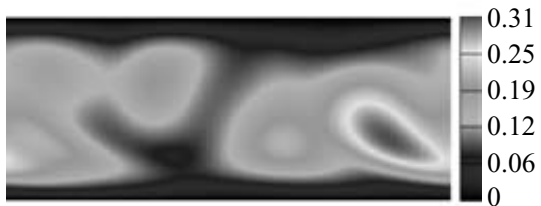

(iv)

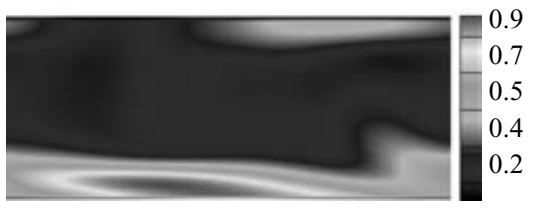

$x$ (b)
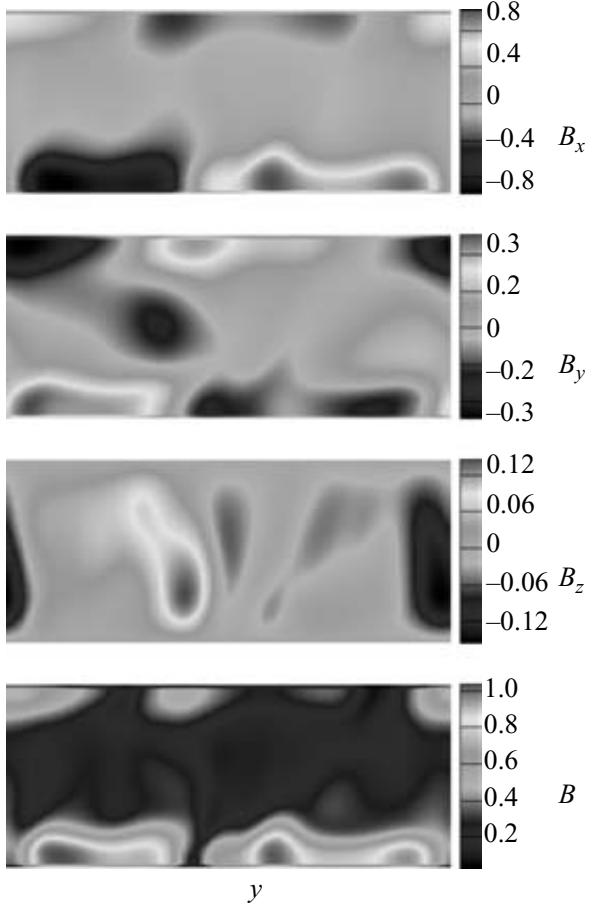

FIGURE 13. Magnetic field for $q=5$ in the dynamical regime. The plots are as in figure 3 .

$\begin{array}{rccrrrr}R e & \tau & q & E_{K} & E_{M} & R m & \left\langle U_{z} \theta\right\rangle \\ 30 & 200 & 50 & 50 & 120 & 500 & 2700 \\ 10 & 200 & 50 & 20 & 100 & 320 & 2400 \\ 0 & 200 & 50 & 35 & 30 & 420 & 3800\end{array}$

TABLE 2. Time-averaged kinetic and magnetic energy at different $R e$, together with magnetic Reynolds number and rate of working of the buoyancy force.

comparison is between $R e=30$ and $R e=0$. These flows are not precisely comparable, as the shear and convection are not entirely independent; however, we observe that the purely convective flow $(R e=0)$ supports a rather weaker magnetic field by comparison with the runs including shear $(R e=10, R e=30)$. In the purely convective case, also, the field is largely trapped against the boundaries, having approximate up-down symmetry, as seen in figures 14 and 15, and observed by Matthews (1998). The perfectly conducting boundary conditions are probably playing an important role here. The purely convective case is also less efficient as a kinematic dynamo, having a growth rate of 0.71 (compared with 3.05 for $R e=50$ ). For $R e=0$, there are no travelling dynamo waves. The middle run, with $R e=10$ shows a low value of the kinetic energy, and supports a strong magnetic field. This confirms that shear is important in generating organized strong magnetic fields and dynamo waves.

\subsection{Runs with varying $R a$ and $\tau$}

We have considered cases with reduced Rayleigh number, which give broadly similar results to the main run discussed above. The only differences emerge when the 

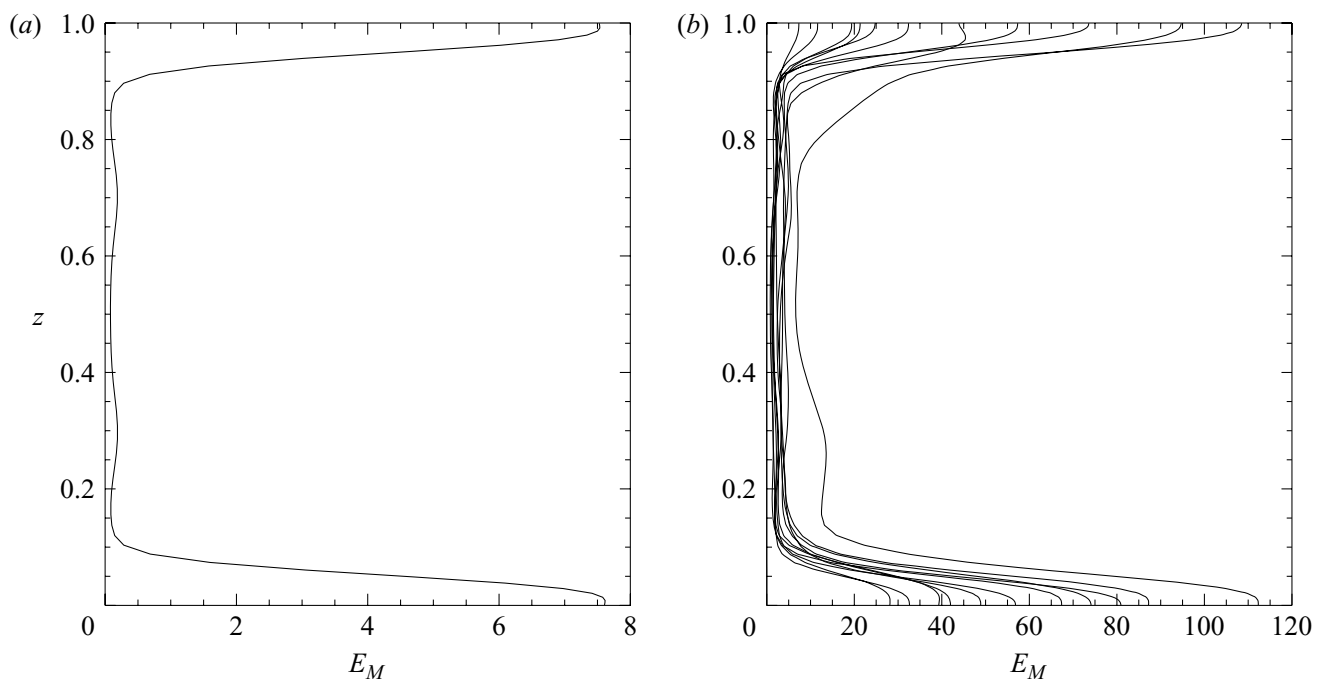

FiguRE 14. Magnetic field profiles $(a)$ in the kinematic regime, $(b)$ at a number of times in the dynamical regime. Plotted is $E_{M}(z)$ (horizontal axis) against $z$ (vertical axis) for $R e=0$.

(a)

(i)

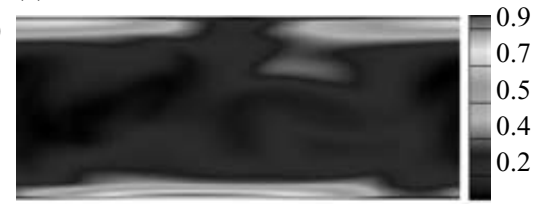

(ii)

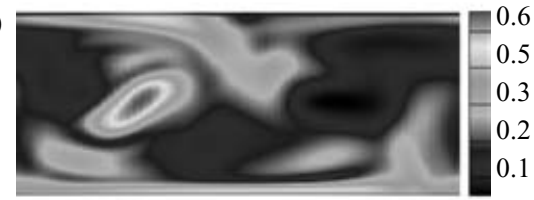

(iii)

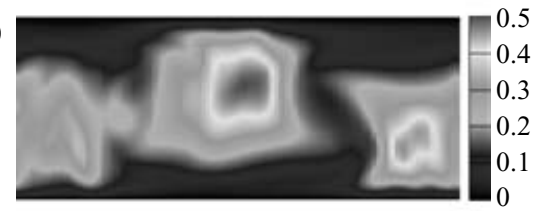

(iv)

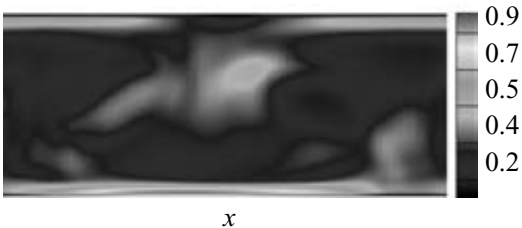

(b)
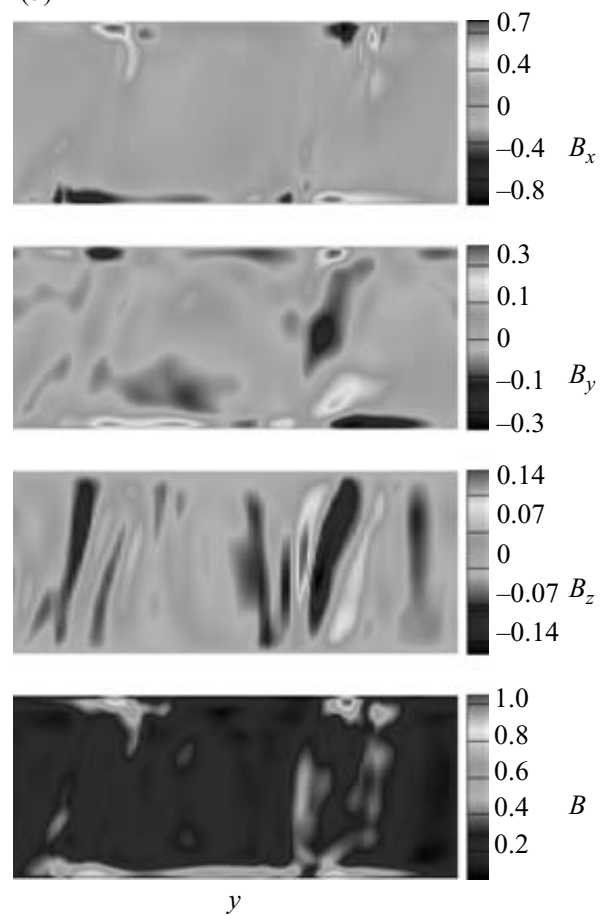

FIGURE 15. Magnetic field with $R e=0$ in the dynamical regime. The plots are as in figure 3 .

Rayleigh number is close to critical, and figures 16 and 17 show the energy in the velocity and magnetic components for $R a=4150 \simeq 1.1 R a_{c}$. Here, the field and the convective components of the flow show a bursty behaviour: as the field becomes 


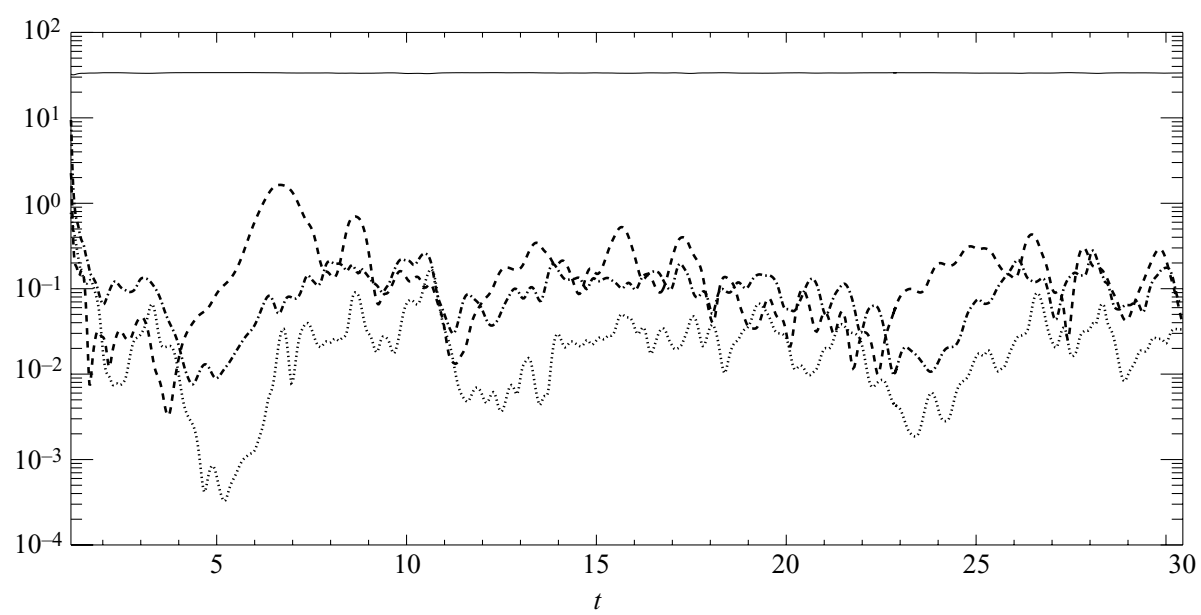

FIGURE 16. The kinetic energy components as in figure 5 for $R a=4125$.

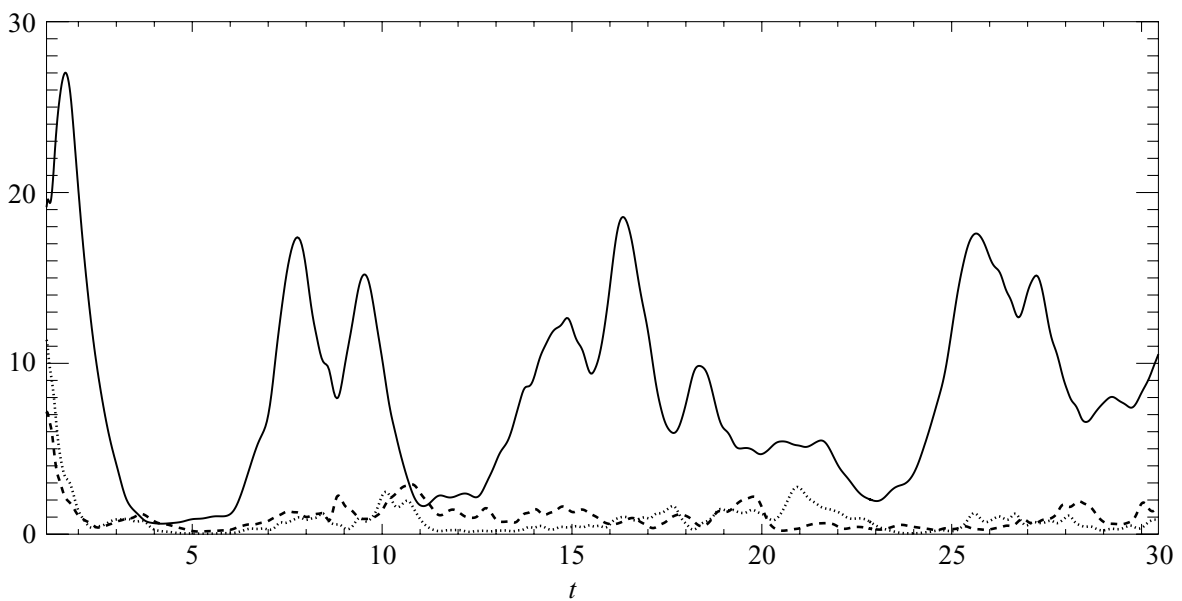

FiguRE 17. Magnetic energies $E_{M(n)}$ as a function of time as in figure 11 for $R a=4125$.

strong, it suppresses the convection, leading to decay of field. This is followed by increase of convection and then increase of field, in an irregular cycle.

Finally, we have considered reducing $\tau$, which has the effect of thickening the shear layer. The kinetic energy in the Ekman-Couette flow is increased, but the flow gradients decrease, giving less intense stretching of the magnetic field. We have not found any great differences when $\tau$ is reduced by half to $\tau=100$. The average kinetic energy is only increased slightly, and in the magnetic field mode $n=1$ is still dominant, but its contribution to the total magnetic energy is smaller.

If $\tau$ is decreased further to 50, the flow field changes its structure, as the broader shear layer now makes rolls with axes aligned with the $x$-axis preferred. The competition between the effects of shear and horizontal component of rotation in aligning convective rolls is explored in Ponty et al. (2003). For this new convective flow the magnetic field decays, and tests using the two-dimensional kinematic dynamo code of Y. Ponty indicate that this flow is not a kinematic dynamo for $q=50$. 


\section{Discussion}

We have studied the saturation of dynamo instabilities in a classical fluid mechanical system, which naturally has the structure of an interface dynamo. There is a thin layer of shear at the base of the plane layer. Above it, and largely unaffected by the shear, a flow is driven by thermal convection, with roll axes controlled by the horizontal component of the rotation of the system. We find that the shear in the system plays an important part in the dynamo process, and that magnetic fields can be understood in terms of $\alpha \omega$-dynamo waves, with the strongest component of field in the shear layer. The clearest link is the phase relationship between the $x$-and $z$-components of field seen in figure 12 for the mode $n=1$ of largest $y$-scale in the system.

Despite this control of the $n=1$ mode, the other modes possess significant amounts of energy (figure 11) and, in fact, the physical space structure of the field shows tubes of field (figure 7 being typical). The tubes have signs modulated by the $n=1$ mode, but the strongest fields have significantly smaller scale. This leads to a key conclusion: an interface dynamo can support magnetic field comprising tubes of small scale, whose sign is modulated on a larger scale. The comparison with the solar dynamo is of interest: at the surface of the Sun we again see thin tubes of field, in terms of sunspots and active regions, but the sign of the field has large-scale correlations given by the Hale polarity laws. Of course, in the solar context there are other mechanisms, not present in our simulations, such as magnetic buoyancy, that can also break up a sheet of field into fine tubes (e.g. Wissink et al. 2000).

We have studied the mechanism for dynamo saturation. We find that the Ekman layer of shear is very robust for the parameter values studied, and the effect of the equilibrating magnetic field is to modify the convection above: in the context of $\alpha \omega$ dynamos the magnetic field equilibrates by quenching the $\alpha$-effect. Note that we have not been able to explore whether this is a result of the modification of the overall chaotic stretching properties of the flow (e.g. Brummell, Cattaneo \& Tobias 2001; Kim et al. 2004) because of the computational expense of following vectors in three dimensions for long enough periods. Of course, in our simulation, the existence of shear has an inevitability about it, as there is a fixed velocity difference of $R e P$ between top and bottom boundaries; however, we also found that the detailed Ekman-layer profile remains essentially unchanged for our runs. In the solar context, there is little evidence of an 11-year cyclic behaviour of the shear in the tachocline (Basu \& Antia 2003; Komm et al. 2003), and so assuming the solar dynamo operates with the benefit of an $\omega$-effect in the tachocline, $\alpha$-quenching is likely to be the most important saturation mechanism. Note, however, that there is evidence of torsional oscillations penetrating deep into the convection zone, and it remains possible that these may affect the tachocline (Vorontsov et al. 2002). One aspect that emerges in our study is the competition between the propagation velocity of $\alpha \omega$ dynamo waves and the perpendicular Ekman flux in the shear flow. In terms of solar modelling, this suggests that the propagation velocity of dynamo waves with fields localized in thin layers of shear could be a delicate matter.

Finally, we considered turning off and on various effects. Increasing the magnetic diffusivity and so decreasing the magnetic Reynolds number gives a similar dynamo with larger-scale magnetic field, equilibrating at lower energies. We observe that turning off the shear, $R e=0$, also leads to a dynamo with lower magnetic energy, fields localized at top and bottom boundaries, and no propagating dynamo wave. Reducing $\tau$ and so thickening the shear layer can lead to a restructuring of the convective cells and switch off the dynamo, at least at the $q$ values we have used. Finally, when $R a$ is reduced to a weakly supercritical value, the dynamo operates 
in an intermittent fashion, with an irregular cycle of dynamo activity followed by damping of convection and damping of magnetic field. Within the context of our model and the limitations of the parameters we can use, shear $R e>0$, convection $R a>0$ and large magnetic Reynolds number, given by $q$, are all instrumental in generating strong coherent magnetic fields.

We should stress again that our model is not intended directly to model the tachocline and solar dynamo. Rather, it is a model that is put together to give a classical fluid system that can function as an interface dynamo, and be explored numerically. The parameters used, for example in (3.1), (3.2), are far from solar values. Indeed, at a latitude of $67.5^{\circ}$ the solar value of $R e$ would be negative; rather, our parameter values were chosen to link to earlier work. Varying $R e$ and $\vartheta$ remains a subject open for further work; we only note that the shear layer is most unstable when $R e$ and $\tau$ have the same sign. Boundary conditions could also be varied, and it may be worthwhile exploring the effects of an electrically insulating medium for $z>1$, modelling the higher magnetic diffusivity present in the convection zone.

In terms of our parameters, if solar molecular values are used for the diffusivities, very large numbers are obtained for $R a, R e$ and $\tau$ (e.g. Ossendrijver 2003), far beyond what can be achieved numerically. Perhaps more importantly, the value of $q$ we have taken is large, whereas the solar value is much smaller, perhaps around $q \sim 10^{-3}$ and the Prandtl number $P \sim 10^{-7}$. The problems of studying dynamos at low $q$ are well-known. Our study, in which the magnetic field is allowed to develop finer structure than the flow, is only one step in the direction of more astrophysically relevant models and parameters.

We are grateful to Dr Yannick Ponty for use of some of his two-dimensional kinematic codes and stability codes, and for valuable discussions. We also benefited from discussions with Chris Jones and Andrew Soward, and from comments of anonymous referees on an earlier draft.

P. Z. gratefully acknowledges support from the Leverhulme Trust under Research Grant F/00144/H. Simulations were run on the PPARC-funded cluster 'saturn', housed in Exeter.

\section{Appendix A}

In our numerical code, we use centred differences on staggered grids to discretize (2.12)-(2.15), with second-order accuracy in space. The pressure is evaluated at points $(i, j, k)$, which may be taken as the centrepoints of a family of cubes. The components of velocity are evaluated at the mid-points of the edges of the cubes, i.e. $u$ at $(i+1 / 2, j, k), v$ at $(i, j+1 / 2, k)$ and $w$ at $(i, j, k+1 / 2)$, and similarly for magnetic field components. Temperature is defined at the corner points of the cubes, i.e. at $(i+1 / 2, j+1 / 2, k+1 / 2)$. The nonlinear terms in (2.12)-(2.15) are approximated by the second-order Adams-Bashforth formula. The Crank-Nicolson scheme is used for the remaining terms. Velocity and temperature are solved first, and then magnetic fields. After discretization, the equations become

$$
\begin{aligned}
& (\Delta t)^{-1}\left(\boldsymbol{U}^{n+1}-\boldsymbol{U}^{n}\right)+\frac{1}{2} \tau P \mathbf{C}\left(\boldsymbol{U}^{n+1}+\boldsymbol{U}^{n}\right) \\
& =-\frac{1}{2} \mathbf{G}\left(\Pi^{n+1}+\Pi^{n}\right)+\frac{1}{2} P\left(\theta^{n+1}+\theta^{n}\right) \boldsymbol{e}_{z}+\frac{1}{2} P \mathbf{L}\left(\boldsymbol{U}^{n+1}+\boldsymbol{U}^{n}\right)+\boldsymbol{f}, \\
& (\Delta t)^{-1}\left(\theta^{n+1}-\theta^{n}\right)=\frac{1}{2} R a\left(U_{z}^{n+1}+U_{z}^{n}\right)+\frac{1}{2} \mathbf{L}\left(\theta^{n+1}+\theta^{n}\right)-g,
\end{aligned}
$$




$$
\begin{gathered}
(\Delta t)^{-1}\left(\boldsymbol{B}^{n+1}-\boldsymbol{B}^{n}\right)=\frac{1}{2}\left(\mathbf{E}^{n+1} \boldsymbol{B}^{n+1}+\mathbf{E}^{n} \boldsymbol{B}^{n}\right)+\frac{1}{2} q^{-1} \mathbf{L}\left(\boldsymbol{B}^{n+1}+\boldsymbol{B}^{n}\right), \\
\mathbf{D} \boldsymbol{U}^{n+1}=0, \quad \mathbf{D} \boldsymbol{B}^{n+1}=0,
\end{gathered}
$$

in which $f$ and $g$ are the Adams-Bashforth discretizations of the nonlinear terms $(\nabla \times \boldsymbol{B}) \times \boldsymbol{B}-(\nabla \times \boldsymbol{U}) \times \boldsymbol{U}$ and $\boldsymbol{U} \cdot \nabla \theta$ calculated using data from times $n-1$ and $n$. $\mathbf{C}, \mathbf{G}, \mathbf{L}$ and $\mathbf{D}$ are discretized operators corresponding to $\widehat{\boldsymbol{\Omega}} \times, \nabla, \nabla^{2}, \nabla \cdot$. Also $\mathbf{E}^{n} \boldsymbol{B}^{n}$ is the discretized form of $\left(\nabla \times\left(\boldsymbol{U}^{n} \times \boldsymbol{B}^{n}\right)\right)$, in which $\mathbf{E}^{n}$ is computed in each time step from the velocity field $\boldsymbol{U}^{n}$.

We solve (A1)-(A4) by adopting a split time-step method (Dukowicz \& Dvinsky 1992), which can be summarized as a two-step scheme: first, we step the NavierStokes and heat equations to obtain the velocity and temperature without the pressure term. The velocity is then corrected by the gradient of pressure, which is obtained by solving a Poisson equation,

$$
\begin{aligned}
&(\mathbf{I}): \quad \widehat{\boldsymbol{u}}=\boldsymbol{U}^{n}-\frac{1}{2} \Delta t \mathbf{G} \Pi^{n}, \\
&\left(1+\frac{1}{2} \Delta t \tau P \mathbf{C}-\frac{1}{2} \Delta t P \mathbf{L}\right) \widetilde{\boldsymbol{u}}-\frac{1}{2} \Delta t P \theta^{n+1} \\
&=\left(1-\frac{1}{2} \Delta t \tau P \mathbf{C}+\frac{1}{2} \Delta t P \mathbf{L}\right) \widehat{\boldsymbol{u}}+\frac{1}{2} \Delta t P \theta^{n}+\Delta t \boldsymbol{f}, \\
&\left(1-\frac{1}{2} \Delta t \mathbf{L}\right) \theta^{n+1}-\frac{1}{2} \Delta t R a \widetilde{u}_{z}=\left(1+\frac{1}{2} \Delta t \mathbf{L}\right) \theta^{n}+\frac{1}{2} \Delta t R a \widehat{u}_{z}-\Delta t g, \\
&(\mathbf{I I}): \quad \mathbf{D G} \Pi^{n+1}=2(\Delta t)^{-1} \mathbf{D} \widetilde{\boldsymbol{u}}, \\
& \boldsymbol{U}^{n+1}=\widetilde{\boldsymbol{u}}-\frac{1}{2} \Delta t \mathbf{G} \Pi^{n+1} .
\end{aligned}
$$

From the above two steps, we update the velocity and then insert it into the induction equation to update magnetic fields. The discretized version of the induction equation does not preserve the condition $\nabla \cdot \boldsymbol{B}=0$; to avoid this problem an artificial scalar gradient $\Pi_{b}$ is introduced (Ramshaw 1983), and a similar split time-step method used.

$$
\begin{aligned}
(\mathbf{I I I}): \quad \widehat{\boldsymbol{b}} & =\boldsymbol{B}^{n}-\frac{1}{2} \Delta t \mathbf{G} \Pi_{b}^{n}, \\
\left(1-\frac{1}{2} \Delta t \mathbf{E}^{n+1}-\frac{1}{2} \Delta t q^{-1} \mathbf{L}\right) \widetilde{\boldsymbol{b}} & =\left(1+\frac{1}{2} \Delta t \mathbf{E}^{n}+\frac{1}{2} \Delta t q^{-1} \mathbf{L}\right) \widehat{\boldsymbol{b}}, \\
(\mathbf{I V}): \quad \mathbf{D G} \Pi_{b}^{n+1} & =2(\Delta t)^{-1} \mathbf{D} \widetilde{\boldsymbol{b}}, \\
\boldsymbol{B}^{n+1} & =\widetilde{\boldsymbol{b}}-\frac{1}{2} \Delta t \mathbf{G} \Pi_{b}^{n+1} .
\end{aligned}
$$

Note that under the perfectly conducting boundary conditions (2.16), the flux of magnetic field in the $x$ - and $y$-directions is constant, zero with our initial conditions. This required an occasional correction to the field to avoid fluxes building up over long runs. The code allows good flexibility in handling boundary conditions for the velocity field, and is parallelized, using 'Aztec' library routines for solving sparse matrix systems. Typical runs involved a spatial resolution of $60 \times 80 \times 60$. The code was tested against results in paper I and Matthews (1998).

\section{Appendix B}

In this Appendix we briefly explore an idealized $\alpha \omega$ model that can be compared with the full three-dimensional runs. A basic model takes the form:

$$
\begin{aligned}
& \left(\partial_{t}+\operatorname{ReP} \Lambda_{2} \partial_{y}-q^{-1} \nabla^{2}\right) B_{x}=\operatorname{ReP} \Lambda_{1}^{\prime} B_{z}, \\
& \left(\partial_{t}+\operatorname{Re} P \Lambda_{2} \partial_{y}-q^{-1} \nabla^{2}\right) B_{z}=-\alpha(z) \partial_{y} B_{x} .
\end{aligned}
$$


The Ekman component (2.20) of the fluid flow is retained and an $\alpha$-effect, $\alpha(z)$ models the convection. Motivated by the form of the helicity (3.15), this could be taken as $\alpha(z)=\alpha_{0} \sin 2 \pi z$, with $\alpha_{0}<0$ (e.g. Moffatt 1978).

This model can be further approximated to give some qualitative information. If we treat $\Lambda_{2}, \Lambda_{1}^{\prime}$ and $\alpha$ as constants ( $\alpha$ being a measure of the alpha effect for low $z$ values), drop the $\nabla^{2}$ term, and take the field proportional to $\mathrm{e}^{\mathrm{i} k y+p t}$ with $k>0$, we obtain a complex growth rate

$$
p= \pm \sqrt{-\mathrm{i} k \alpha \operatorname{ReP} \Lambda_{1}^{\prime}}-\mathrm{i} k \operatorname{Re} P \Lambda_{2} \text {. }
$$

Note that $k$ corresponds to $n k_{y}$ in the full numerical simulation. A growing mode has real growth rate

$$
\gamma \equiv \operatorname{Re} p=\sqrt{\frac{1}{2} k\left|\alpha \operatorname{ReP} \Lambda_{1}^{\prime}\right|}
$$

and phase speed

$$
c \equiv-k^{-1} \operatorname{Im} p=\sqrt{\frac{1}{2} k^{-1}\left|\alpha \operatorname{Re} P \Lambda_{1}^{\prime}\right|} \operatorname{sign}\left(\alpha \operatorname{Re} P \Lambda_{1}^{\prime}\right)+\operatorname{Re} P \Lambda_{2} .
$$

Here we see clearly the role of the second, Ekman flux term in carrying the field in the $y$-direction. For our model we have

$$
\Lambda_{1}^{\prime}<0, \quad \Lambda_{2}<0, \quad \alpha<0, \quad \operatorname{Re}>0,
$$

and so we see that the velocity of the $\alpha \omega$-dynamo wave (first term) is in the $+y$ direction, while the Ekman flux advection is in the $-y$-direction. There is competition between the two to determine the overall velocity of the dynamo wave (Yoshimura 1975; Dikpati \& Gilman 2001). Note that this would remain the case even if we reverse the direction of the underlying shear flow, changing the sign of $U_{0}$ and so of only $R e$ in (B 6). There is a phase shift between the magnetic field components of

$$
\arg \left(B_{z} / B_{x}\right)=(\pi / 4) \operatorname{sign}\left(\alpha \operatorname{Re} \Lambda_{1}^{\prime}\right)-(\pi / 2) \operatorname{sign} \alpha .
$$

Similar results are obtained when the full model (B1)-(B2) is solved numerically.

This highly simplified modelling makes contact with our dynamo simulation in two ways. First, the phase shift between $x$ - and $z$-directed field is given by $3 \pi / 4 \simeq 2.36$ which is approximately in line with the results in figure 12(c). Secondly, there is evidence that the dynamo waves in our main simulation involve a competition between Ekman pumping velocity (given by $\max _{z} U_{E k, y}=-0.3224 R e P \simeq-9.67$ ) and an $\alpha \omega$ dynamo wave velocity. The reason is that for the main run, in kinematic regimes the magnetic field has a phase velocity $c_{1} \simeq-1.5$ whereas in the equilibrated regime $c_{1} \simeq-4$, which is consistent with suppression of the alpha effect (via suppression of the convection observed) and so the $\alpha \omega$ dynamo velocity in (B5).

\section{REFERENCES}

Basu, S. \& Antia, H. M. 2003 Changes in solar dynamics from 1995 to 2002. Astrophys. J. 585, $533-565$.

Brandenburg, A., Nordlund, A., Pulkkinen, P., Stein, R. F. \& Tuominen, I. 1990 3D simulation of turbulent cyclonic magnetoconvection. Astron. Astrophys. 232, 277-291.

Brandenburg, A., Nordlund, A., Stein, R. F. \& Torkelsson, U. 1995 Dynamo generated turbulence and large scale magnetic fields in a Keplerian shear flow. Astrophys. J. 446, 741-754.

Brown, T. M., Christensen-Dalsgand, J., Dziembowski, W. A., Goode, P. R., Gough, D. O. \& MorRow, C. 1989 Inferring the Sun's internal angular velocity from observed p-mode frequency splittings. Astrophys. J. 343, 526-546. 
Brummell, N. H., Cattaneo, F. \& Tobias, S. M. 2001 Linear and nonlinear dynamo properties of time-dependent ABC flows. Fluid Dyn. Res. 28, 237-265.

Busse, F. H. 1975 A model of the geodynamo. Geophys. J. R. Astr. Soc. 42, 437-459.

Busse, F. H. 2002 Convective flows in rapidly rotating spheres and their dynamo action. Phys. Fluids 14, 1301-1314.

Cattaneo, F., Hughes, D. W. \& Thelen, J. C. 2002 The nonlinear properties of a large-scale dynamo driven by helical forcing. J. Fluid Mech. 456, 219-237.

Childress, S. \& Gilbert, A. D. 1995 Stretch, twist, fold: the fast dynamo. Lecture Notes in Physics, Springer.

Childress, S. \& Soward, A. M. 1972 Convection driven hydromagnetic dynamo. Phys. Rev. Lett. 29, 837-839.

Cline, K. S., Brummell, N. H. \& Cattaneo, F. 2003 Dynamo action driven by shear and magnetic buoyancy. Astrophys. J. 599, 1449-1468.

Deluca, E. E. \& Gilman, P. A. 1986 Dynamo theory for the interface between the convection zone and the radiative interior of a star. Part I: Model equations and exact solutions. Geophys. Astrophys. Fluid Dyn. 37, 85-127.

Deluca, E. E. \& Gilman, P. A. 1988 Dynamo theory for the interface between the convection zone and the radiative interior of a star. Part II: Numerical solutions of the nonlinear equations. Geophys. Astrophys. Fluid Dyn. 43, 119-148.

Dikpati, M. \& Gilman, P. A. 2001 Flux-transport dynamos with $\alpha$-effect from global instability of tachocline differential rotation: a solution for magnetic parity selection in the Sun. Astrophys. J. 559, 428-442.

Dukowicz, J. K. \& Dvinsky, A. S. 1992 Approximate factorization as a high-order splitting for the implicit incompressible-flow equations. J. Comput. Phys. 102, 336-347.

Dziembowski, W., Goode, P. H. \& Libbrecht, K. G. 1989 The radial gradient in the Sun's rotation. Astrophys. J. 337, L53-L57.

Garaud, P. 2002 Dynamics of the solar tachocline. I. An incompressible study. Mon. Not. R. Astr. Soc. 329, 1-17.

Gilbert, A. D. 2003 Dynamo theory. In Handbook of Mathematical Fluid Dynamics (ed. S. Friedlander \& D. Serre), vol. 2, pp. 355-441. Elsevier.

Gilman, P. A. 1983 Dynamically consistent nonlinear dynamos driven by convection in a rotating spherical shell. II. Dynamos with cycles and strong feedbacks. Astrophys. J. Suppl. 53, 243-268.

Glatzmaier, G. A. 1984 Numerical simulations of stellar convective dynamos. I. The model and method. J. Comput. Phys. 55, 461-484.

Glatzmaier, G. A. 1985a Numerical simulations of stellar convective dynamos. II. Field propagation in the convection zone. Astrophys. J. 291, 300-307.

Glatzmaier, G. A. $1985 b$ Numerical simulations of stellar convective dynamos. III. At the base of the convection zone. Geophys. Astrophys. Fluid Dyn. 31, 137-150.

Goode, P. R., Dziembowski, W. A., Korzennik, S. G. \& Rhodes, E. J. 1991 What we know about the Sun's internal rotation from solar oscillations. Astrophys. J. 367, 649-657.

Gough, D. O. \& McIntyre, M. E. 1998 Inevitability of a magnetic field in the Sun's radiative interior. Nature 394, 755-757.

Gough, D. O., SekiI, T. \& Stark, P. B. 1996 Inferring spatial variation of solar properties from helioseismic data. Astrophys. J. 459, 779-791.

Griffiths, G. L., Bassom, A. P., Soward, A. M. \& Kuzanyan, K. M. 2001 Nonlinear $\alpha^{2} \Omega$-dynamo waves in stellar shells. Geophys. Astrophys. Fluid Dyn. 94, 85-133.

Hoffman, N., Busse, F. H. \& Chen, W.-L. 1998 Transition to complex flows in the Ekman-Couette layer. J. Fluid. Mech. 366, 311-331.

Jones, C. A. \& Roberts, P. H. 2000 Convection driven dynamos in a rotating plane layer. J. Fluid Mech. 404, 311-343.

Kim, E.-J., Hughes, D. W. \& Soward, A. M. 1999 An investigation into high conductivity dynamo action driven by rotating convection. Geophys. Astrophys. Fluid Dyn. 91, 303-332.

Kim, E.-J., Hughes, D. W. \& Soward, A. M. 2004 A nonlinear dynamo driven by rapidly rotating convection. Geophys. Astrophys. Fluid Dyn. 98, 325-343.

Komm, R., Howe, R., Durney, B. R. \& Hill, F. 2003 Temporal variation of angular momentum in the solar convection zone. Astrophys. J. 586, 650-662. 
Krause, F. \& RäbleR, K.-H. 1980 Mean-Field Magnetohydrodynamics and Dynamo Theory. Pergamon.

Matthews, P. C. 1998 Dynamo action in simple convective flows. Proc. R. Soc. Lond. A 455, 1829-1840.

Matthews, P. C. \& Cox, S. 1997 Linear stability of rotating convection in an imposed shear flow. J. Fluid Mech. 350, 271-293.

Moffatt, H. K. 1978 Magnetic Field Generation in Electrically Conducting Fluids. Cambridge University Press.

Nordlund, A., Brandenburg, A., Jennings, R.L., Rieutord, M., Ruokolainen, J., Stein, R. F. \& Tuominen, I. 1992 Dynamo action in stratified convection with overshoot. Astrophys. J. 392, $647-652$.

Ossendrijver, M. 2003 The solar dynamo. Astron. Astrophys. Rev. 11, 287-367.

Parker, E. N. 1979 Cosmical Magnetic Fields. Clarendon.

PARKer, E. N. 1993 A solar dynamo surface wave at the interface between convection and nonuniform rotation. Astrophys. J. 408, 707-719.

Ponty, Y., Gilbert, A. D. \& Soward, A. M. 2001 Kinematic dynamo action in large magnetic Reynolds number flows driven by shear and convection. J. Fluid Mech. 435, 261-287. (Referred to herein as paper I.)

Ponty, Y., Gilbert, A. D. \& Soward, A. M. 2003 The onset of thermal convection in EkmanCouette shear flow with oblique rotation. J. Fluid Mech. 487, 91-123.

Prautzsch, T. 1993 The dynamo mechanism in the deep convection zone of the Sun. In Solar and planetary dynamos (ed. M. R. E. Proctor, P. C. Matthews \& A. M. Rucklidge), pp. 249-256. Cambridge University Press.

Ramshaw, J. D. 1983 A method for enforcing the solenoidal condition on magnetic field in numerical calculations. J. Comput. Phys. 52, 592-596.

RoALD, C. B. 1998 A two-layer alpha-omega dynamo model with dynamic feedback on the omegaeffect. Mon. Not. R. Astr. Soc. 300, 397-410.

Roberts, P. H. 1992 The dynamo mechanism. In Production et Rôle du Champ Magnetique, pp. 61-117. Observatoire de Paris, Section de Meudon.

Rotvig, J. \& Jones, C. A. 2002 Rotating convection driven dynamos at low Ekman number. Phys. Rev. E 66, 056308-1-15.

Rüdiger, G. \& BRANDEnburg, A. 1995 A solar dynamo in the overshoot layer - cycle period and butterfly diagram. Astron. Astrophys. 296, 557-566.

St Pierre, M. G. 1993 The strong field branch of the Childress-Soward dynamo. In Solar and Planetary Dynamos (ed. M. R. E. Proctor, P. C. Matthews \& A. M. Rucklidge), pp. 295-302. Cambridge University Press.

Spiegel, E. A. \& Zahn, J.-P. 1992 The Solar tachocline. Astron. Astrophys. 265, 106-114.

Thelen, J. C. 2000 Non-linear alpha omega-dynamos driven by magnetic buoyancy. Mon. Not. R. Astr. Soc. 315, 165-183.

Tobias, S. M. 1996 Diffusivity quenching as a mechanism for Parker's surface dynamo. Astrophys. J. 467, 870-880.

Tobias, S. M., Brummell, N. H., Clune, T. L. \& Toomre, J. 2001 Transport and storage of magnetic field by overshooting turbulent compressible convection. Astrophys. J. 549, 1183-1203.

Tobias, S. M., Proctor, M. R. E. \& KNobloch, E. 1998 Convective and absolute instabilities of fluid flows in finite geometry. Physica D 113, 43-72.

Vorontsov, S. V., Christensen-Dalsgaard, J., Schou, J., Strakhov, V. N. \& Thompson, M. J. 2002 Helioseismic measurement of Solar torsional oscillations. Science 296, 101-103.

Weiss, N. O. 1994 Solar and stellar dynamos. In Lectures on Solar and Planetary Dynamos (ed. M. R. E. Proctor \& A. D. Gilbert), pp. 59-95. Cambridge University Press.

Wissink, J. G., Matthews, P. C., Hughes, D. W. \& Proctor, M. R. E. 2000 The three-dimensional breakup of a magnetic layer. Mon. Not. R. Astr. Soc. 318, 501-510.

Yoshimura, H. 1975 Solar cycle dynamo wave propagation. Astrophys. J. 201, 740-748.

Zhang, K., Chan, K. H., Zou, J., LiaO, X. \& Schubert, G. 2003 A three-dimensional spherical nonlinear interface dynamo. Astrophys. J. 596, 663-679. 